\title{
ON AUTOMORPHISMS OF LIE ALGEBRAS OF CLASSICAL TYPE
}

\author{
BY
}

\author{
GEORGE B. SELIGMAN
}

In two recent papers $\left(^{1}\right)([12]$ and [13], the latter hereafter referred to as (C)), W. H. Mills and the author have developed a structure theory for certain Lie algebras over arbitrary fields of characteristic different from 2 and 3. This class of algebras is defined by axioms in (C), and its members will be referred to as Lie algebras of classical type. They are closely related to semisimple Lie algebras over algebraically closed fields of characteristic zero, not only in that such algebras are of classical type, but also in that nearly every Lie algebra of classical type may be obtained from such an algebra of characteristic zero by reducing its integral structure constants modulo a prime and performing a suitable extension of the prime field $[2 ; 3 ; 4 ; 15]$.

There are several reasons for seeking information about the automorphisms of these Lie algebras. In case the base field $\mathfrak{F}$ is finite, recent investigations by Chevalley [2] have revealed new simple finite groups closely related to the automorphism groups of the "exceptional" simple Lie algebras of classical type over $\mathfrak{F}$. It would be of interest to obtain more precise information as to the relations between these groups. If one is to make an analysis of all normal simple Lie algebras over arbitrary fields of characteristic different from 2 or 3 which become simple Lie algebras of classical type when one passes to the algebraic closure (e.g., normal simple algebras with nondegenerate Killing forms), the techniques used in the past require a knowledge of the automorphisms in the algebraically closed case $[9 ; 10 ; 11 ; 17]$. Since the groups which occur as automorphism groups include several of the classical groups our viewpoint represents an alternate, if perhaps devious, method for getting information about the classical groups. In the case of the automorphism groups of the exceptional algebras, the scarcity of knowledge on these linear groups lends interest to any pertinent results. It is proposed to discuss the meaning of our results for the various linear groups in a later paper.

The techniques utilized are basically of two kinds. First, the analogy of simple Lie algebras of classical type with simple Lie algebras over algebraically closed fields of characteristic zero is refined so as to verify a number of results on roots and the Weyl group in the general case. This procedure, which is carried out in II, was developed for Lie algebras with nondegenerate Killing forms by Curtis $[3 ; 4]$, and our techniques are basically the same

Presented to the Society, November 22, 1958 under the title On the group of invariant automorphisms of a Lie algebra; received by the editors June 10, 1958.

(1) Numbers in brackets refer to the bibliography at the end of the paper. 
as his. Secondly, in III and IV the transcendental techniques of Gantmacher with exponentials [7] are replaced by the use of iterated exponentials which are defined over all fields of characteristic different from 2 and 3. These mappings have been discussed from a somewhat different viewpoint by Chevalley [2]. Considerable similarity with his techniques will be noted. The results on the situation of the group of invariant [15] automorphisms in the full automorphism group in the algebraically closed case are stated in V.

I. Automorphisms and standard Cartan subalgebras. Let $\&$ be a Lie algebra of classical type, in the sense of (C). Let $\sigma$ be an automorphism of $\mathfrak{R}$ and $\mathfrak{S}$ an abelian Cartan subalgebra relative to which (iii) $-(\mathrm{v})$ of $(\mathrm{C})$ are satisfied (such a Cartan subalgebra will in the sequel be called a standard Cartan subalgebra). Then $\mathfrak{S} \sigma$ is again an abelian Cartan subalgebra of $\mathfrak{l}$, and if $\mathfrak{R}=\mathfrak{S}+\sum_{\alpha} \mathfrak{R}_{\alpha}$ is the Cartan decomposition relative to $\mathfrak{S}$, we have $\mathbb{R}=\mathfrak{S} \sigma+\sum_{\alpha} \mathfrak{R}_{\alpha} \sigma$, again a vector space direct sum. Moreover, if $e_{\alpha} \in \mathfrak{R}_{\alpha}$, then $\left[e_{\alpha} \sigma, h \sigma\right]=\left[e_{\alpha} h\right] \sigma=\alpha(h) e_{\alpha} \sigma$ for all $h \in \mathfrak{S}$; that is, $\ell_{\alpha} \sigma$ is a root-space relative to $\mathfrak{S} \sigma$, and the corresponding root is $\alpha^{\sigma}: \alpha^{\sigma}(h \sigma)=\alpha(h)$. Thus $\mathfrak{R}=\mathfrak{S} \sigma+\sum_{\alpha} \mathfrak{R}_{\alpha} \sigma$ is the Cartan decomposition of $\mathfrak{R}$ relative to $\mathfrak{S} \sigma$. Also $\left[\mathfrak{R}_{\alpha} \sigma, \mathfrak{R}_{-\alpha} \sigma\right]=\left[\mathfrak{R}_{\alpha}, \mathfrak{R}_{-\alpha}\right] \sigma$ is one-dimensional, and if $\alpha^{\sigma}$ is a nonzero root relative to $\mathfrak{g} \sigma$ and $\beta \in(\mathfrak{D} \sigma)^{*}$, then $\beta=\gamma^{\sigma}$, where $\gamma \in \mathfrak{S}^{*}$ and $\beta(h \sigma)=\gamma(h)$ for all $h \in \mathfrak{S}$. Since $\gamma \rightarrow \gamma^{\sigma}$ is evidently a linear mapping of $\mathfrak{S}^{*}$ onto $(\mathfrak{S} \sigma)^{*}$, we see that if all the forms $\beta+m \alpha^{\sigma}$ $=(\gamma+m \boldsymbol{\alpha})^{\sigma}(m$ an integer $)$ were roots relative to $\mathfrak{S} \sigma$, then all the forms $\gamma+m \boldsymbol{\alpha}$ would be roots relative to $\mathfrak{S}$, contrary to (v). Thus (iii)-(v) of (C) are satisfied by $\mathbb{\Omega}$ relative to $\mathfrak{S} \sigma ; \mathfrak{S} \sigma$ is a standard Cartan subalgebra.

Now $\mathfrak{l}=\mathfrak{R}_{1} \oplus \cdots \oplus \mathfrak{R}_{\mathfrak{s}}$, where the $\mathfrak{R}_{i}$ are nonabelian simple ideals satisfying the same axioms as $\&$ does $((\mathrm{C})$, Theorem 4$)$. Then it is well known that each $\mathfrak{R}_{i} \sigma$ is an $\mathfrak{R}_{j}$, i.e., $\sigma$ permutes the $\mathfrak{R}_{i}$. Of course, $\mathfrak{R}_{i} \sigma=\mathfrak{R}_{j}$ is only possible if $\mathfrak{R}_{i}$ and $\mathfrak{R}_{j}$ are isomorphic simple algebras. If we collect all the isomorphic $\mathfrak{R}_{i}$ in the decomposition $\mathfrak{R}=\Omega_{1} \oplus \cdots \oplus \Omega_{s}$ into blocks, then $\sigma$ effects a permutation in each block. We cannot hope to achieve this permutation through operations generated by the Lie product in the algebra, since these will always map each $\mathfrak{R}_{i}$ into itself. However, after performing one of the finite set of all possible permutations of the $\mathfrak{R}_{i}$ in each block, we can replace $\sigma$ by an automorphism which maps each $\mathfrak{R}_{i}$ into itself. In this sense the problem of describing all automorphisms of $\mathfrak{R}$ is reduced to the case where $\mathfrak{R}$ is simple.

We therefore assume $\mathfrak{R}$ is simple, we let $\mathfrak{S}$ be as above, and we let $\alpha_{1}, \cdots$, $\alpha_{r}$ be a fundamental system of roots relative to $\mathfrak{S}$. That is, $\alpha_{1}, \cdots, \alpha_{r}$ span $\mathfrak{S}^{*}, \alpha_{i}-\alpha_{j}$ is not a root if $i \neq j$, and the $r \times r$ matrix $\left(A_{i j}\right)$ of rational integers ((C), (xiv) and $\S 7)$ is nonsingular; moreover, $\left(A_{i j}\right)$ is ir tecomposable: for every $i \neq j$, there lare indices $i_{0}=i, i_{1}, i_{2}, \cdots, i_{k}=j$ such that $A_{i_{\nu}, i_{v+1}} \neq 0$, $0 \leqq \nu \leqq k-1$. Finally, there is a unique root $\beta$ relative to $\mathfrak{S}$ such that no $\beta-\alpha_{i}$ is a root, $1 \leqq i \leqq r((C), \S 10)$. Since the roots relative to $\mathfrak{S} \sigma$ span $(\mathfrak{S} \sigma)^{*}$ and since $\alpha \rightarrow \alpha^{\sigma}$ is linear from $\mathfrak{S}^{*}$ onto $(\mathfrak{S} \sigma)^{*}$ and maps the set of roots relative to $\mathfrak{S}$ onto that relative to $\mathfrak{5} \sigma$, it follows that $\alpha_{1}^{\sigma}, \cdots, \alpha_{r}^{\sigma} \operatorname{span}(\mathfrak{S} \sigma)^{*}$. 
If $\alpha_{i}^{\sigma}-\alpha_{j}^{\sigma}$ were a root for some $i \neq j$, then so would be $\left(\alpha_{i}^{\sigma}-\alpha_{i}^{\sigma}\right)^{\sigma^{-1}}=\alpha_{i}-\alpha_{j}$, a contradiction. The integer $A_{i j}=A_{\alpha_{i}, \alpha_{j}}$ is determined by the conditions that $A_{i i}=2$, and if $i \neq j,-A_{i j}=m-1$, where $m$ is the first non-negative integer such that $\alpha_{i}+m \alpha_{j}$ is not a root. Now $A_{\alpha_{i}, \alpha_{i}^{\sigma}}^{\sigma}=2$ by definition, and $-A_{\alpha_{i}, \alpha_{j}^{\sigma}}^{\sigma}$ $=t-1$, where $t$ is the first non-negative integer such that $\alpha_{i}^{\sigma}+t \alpha_{j}^{\sigma}$ is not a root. But $\alpha_{i}+m \alpha_{j}$ is a root if and only if $\left(\alpha_{i}+m \alpha_{j}\right) \sigma=\alpha_{i}^{\sigma}+m \alpha_{j}^{\sigma}$ is a root relative to $\mathfrak{S E}_{\mathrm{C}}$. Thus $m=t$ and $A_{\alpha_{i}^{\sigma}, \alpha_{j}^{\sigma}}=A_{i j}$, so that $\left(A_{\alpha_{i}^{\sigma}, \alpha_{j}^{\sigma}}\right)$ is nonsingular and indecomposable. Finally, $\beta^{\sigma}$ is the unique minimal root relative to $\alpha_{1}^{\sigma}, \cdots, \alpha_{r}^{\sigma}$, in the sense in which $\beta$ is the unique minimal root relative to $\alpha_{1}, \cdots, \alpha_{r}$. Therefore $\alpha_{1}^{\sigma}, \cdots, \alpha_{r}^{\sigma}$ constitute a fundamental system of roots relative to $\mathfrak{S} \sigma$, and $\left(A_{\alpha_{i}, \alpha_{j}^{\sigma}}^{\sigma}\right)=\left(A_{i j}\right)$.

If $e_{\alpha} \neq 0$ is a root-vector $(\alpha \neq 0)$ relative to some standard Cartan subalgebra $\mathfrak{F}$ of $\mathfrak{R}$, then $\operatorname{ad}\left(e_{\alpha}\right): x \rightarrow\left[x e_{\alpha}\right]$ is a derivation $D$ of $\mathfrak{R}$, and $D^{4}=0$ (see [15]). Since the characteristic of $\mathfrak{F}$ is not 2 or $3, A=\exp (\lambda D)$ is defined by the exponential power series for each $\lambda \in \mathfrak{F}$, and $A$ is an automorphism of $\mathfrak{R}$. For $p=5$ and $\mathfrak{R}$ of type $G_{2}$, a special treatment is required to show that $A$ is an automorphism; in all cases other than $G_{2}, D^{3}=0$ and no special argument is required. The group of automorphisms generated by all these $\exp \left(\lambda\right.$ ad $\left.\left(e_{\alpha}\right)\right)$, where $\lambda$ runs through $\mathfrak{F}$ and where $e_{\alpha}$ runs through a set of nonzero root-vectors belonging to all nonzero roots relative to all standard Cartan subalgebras $\mathfrak{S}$ of $\mathbb{R}$, will be called the group of invariant automorphisms of $\mathfrak{R}$, and will be denoted by $\mathfrak{\Im}$. In [15] we have shown that if $\mathfrak{F}$ is algebraically closed, and if $\mathfrak{S}_{1}$ and $\mathfrak{F}_{2}$ are any two standard Cartan subalgebras of $\mathfrak{R}$, then there is an invariant automorphism $A$ such that $\mathfrak{S}_{1} A=\mathfrak{S}_{2}$. In particular, if $\mathfrak{S}_{\mathfrak{S}}$ is a standard Cartan subalgebra and $\sigma$ is an automorphism of $\&$, then $\mathfrak{S} \sigma=\mathfrak{S} A$ for some invariant automorphism $A$, and $\mathfrak{5} \sigma A^{-1}=\mathfrak{S}$. Thus, in the algebraically closed case, the use of invariant automorphisms reduces the study of automorphisms to the investigation of those automorphisms which map a certain standard Cartan subalgebra onto itself.

Let us summarize the results of this section.

Theorem 1. Let $\mathbb{R}$ be a simple Lie algebra of classical type, $\mathfrak{S}$ a standard Cartan subalgebra of $\mathbb{R}$, and $\alpha_{1}, \cdots, \alpha_{r}$ a fundamental system of roots relative to $\mathfrak{S}$. Let $\sigma$ be an automorphism of $\mathbb{R}$. Then $\mathfrak{S} \sigma$ is a standard Cartan subalgebra of $\mathfrak{R}$, and if $\alpha^{\sigma}$ is defined by $\alpha^{\sigma}(h \sigma)=\alpha(h)$ for each $\alpha \in \mathfrak{S}^{*}$, then $\alpha^{\sigma}$ is a root relative to $\mathfrak{S} \sigma$ if and only if $\alpha$ is a root relative to $\mathfrak{S}$. The mapping $\alpha \rightarrow \alpha^{\sigma}$ is a linear isomorphism of $\mathfrak{S}^{*}$ onto $(\mathfrak{S} \sigma)^{*}$, and $\alpha_{1}^{\sigma}, \cdots, \alpha_{r}^{\sigma}$ form a fundamental system of roots relative to $\mathfrak{S}_{\sigma} \sigma$ with $A_{\alpha_{i}, \alpha_{j}{ }^{\sigma}}=A_{\alpha_{i}, \alpha j}$ for all $i, j$. If the base field is algebraically closed, there is an invariant automorphism $A$ of $\mathbb{R}$ such that $\mathfrak{5} \sigma=\mathfrak{5} A$.

II. Fundamental systems and the Weyl group. Let $\mathfrak{l}, \mathfrak{S}$, and $\alpha_{1}, \cdots, \alpha_{r}$ be as in Theorem 1. For each $i, 1 \leqq i \leqq r$, let $h_{i}$ be that unique element of $\left[\mathfrak{R}_{-\alpha_{i}}, \mathfrak{R}_{\alpha_{i}}\right]$ such that $\alpha_{i}\left(h_{i}\right)=2(=2 \cdot 1$, where 1 is the unity of $\mathfrak{F})$. That such a choice is possible and unique follows from (iv) and (xi) of (C). We define 
a linear transformation $S_{\alpha_{i}}=S_{i}$ of $\mathfrak{S}^{*}$ by $\phi S_{i}=\phi-\phi\left(h_{i}\right) \alpha_{i}, \phi \in \mathfrak{S}^{*}$. Evidently $S_{i}^{2}=I$, the identity transformation. If $\alpha$ is any root, then

$$
\alpha S_{i}=\alpha-\alpha\left(h_{i}\right) \alpha_{i}=\alpha-\bar{A}_{\alpha, i} \alpha_{i}=\alpha-A_{\alpha, i} \alpha_{i},
$$

where $\bar{n}$ is the residue class, in the prime field of $\mathfrak{F}$, of the rational integer $n$. This equation follows from (xiv) of (C). But $\alpha-A_{\alpha, i} \alpha_{i}$ is a root, by the definition of $A_{\alpha, i}=A_{\alpha, \alpha_{i}}$ in (xiv) of (C); therefore each $S_{i}$ permutes the roots relative to $\mathfrak{S}$. Since the roots span $\mathfrak{S}^{*}$, each $S_{i}$ is completely determined by its effect on the roots alone. Thus the group $\mathfrak{W}$ generated by the $S_{i}$ may be regarded as a subgroup of the group of all permutations of the set of roots relative to $\mathfrak{S}$, and so $\mathfrak{W}$ is a finite group. As in Theorem 16.1 of [16], we could show directly that $\mathfrak{R}$ is independent of the chosen fundamental system $\alpha_{1}, \cdots, \alpha_{r}$. However, this result will be a corollary of the result in [16] after we have proved Lemma 5 below. Meanwhile we shall anticipate this independence by referring to $\mathfrak{B}$ simply as the Weyl group of $\mathfrak{\imath}$ relative to $\mathfrak{S}$.

Next we shall establish for Lie algebras of classical type a certain one-toone correspondence between the elements of the Weyl group and fundamental systems of roots relative to $\mathfrak{S}$. Following an idea developed by Curtis (Proposition 5 of [4]) for algebras with nondegenerate Killing forms, we shall achieve this objective by setting up an "isomorphism" betineen the sets of roots of two simple Lie algebras of classical type, perhaps over base fields of different characteristics, provided these two Lie algebras have the same matrix $\left(A_{i j}\right)$ for certain fundamental systems. There is also associated with this identification an isomorphism of the respective Weyl groups as transformation groups acting on the roots. The desired results for? are then immediate from the corresponding ones for simple algebras over algebraically closed fields of characteristic zero.

Again let $\mathbb{Z}$ be a simple Lie algebra of classical type, $\mathfrak{5}$ a standard Cartan subalgebra, $\alpha_{1}, \cdots, \alpha_{r}$ a fundamental system of roots relative to $\mathfrak{H}$. Then there is a unique minimal root $\delta$ relative to $\alpha_{1}, \cdots, \alpha_{r}$, in the sense of $I$, and if $\beta$ is any root, there is a sequence $\beta, \beta-\alpha_{i_{1}}, \beta-\alpha_{i_{1}}-\alpha_{i_{2}}, \cdots, \beta-\alpha_{i_{1}}-\ldots$ $-\alpha_{i_{k}}=\delta$ of roots, $1 \leqq i_{j} \leqq r$ ( 0 is here regarded as a root). The non-negative rational integer $k=N(\beta)$ is uniquely determined by $\beta$, and the integer $L(\beta)$ $=N(\beta)-N(0)$ is called the level of $\beta$. (These and the following are the results of $\S 11$ of $(\mathrm{C}))$. The function $L$ has the properties:

(a) $L(\beta)=0$ if and only if $\beta=0$.

(b) $L(\beta)=1$ if and only if $\beta=\alpha_{i}$ for some $i ; L(\beta)=-1$ if and only if $\beta=-\alpha_{i}$ for some $i$.

(c) If $L(\beta)>0$, then $\beta-\alpha_{i}$ is a root for some $i$; if $L(\beta)<0$, then $\beta+\alpha_{i}$ is a root for some $i$.

(d) If $\alpha, \beta$, and $\alpha+\beta$ are roots, then $L(\alpha+\beta)=L(\alpha)+L(\beta)$.

(e) $L(-\beta)=-L(\beta)$ for all roots $\beta$.

We shall also need: 
(f) The matrix $\left(A_{i j}\right)$ is nonsingular $(\$ \S 10$ and 8 of $(\mathrm{C}))$ and:

(g) If $\alpha, \beta, \gamma, \cdots, \rho, \zeta$ are roots relative to $\mathfrak{S}$, and if $\alpha+\beta, \alpha+\beta+\gamma, \cdots$, $\alpha+\beta+\cdots+\rho$ are roots, then if $\zeta \neq 0, A_{\alpha+\beta+\cdots+\rho, \zeta}=A_{\alpha, \zeta}+\cdots+A_{\rho, \zeta}$ (Lemma 3 of (C), Lemmas D and L of [12]).

LemMa 1. Every root $\beta$ with $L(\beta)>0$ is a term of a chain $\alpha_{i_{1}}, \alpha_{i_{1}}+\alpha_{i_{2}}, \cdots$, $\alpha_{i_{1}}+\alpha_{i_{2}}+\cdots+\alpha_{i_{s}}=\beta\left(1 \leqq i_{j} \leqq r\right)$ where all terms are roots. The number $t(\beta, i)$ of times which $\alpha_{i}$ occurs in the sum $\alpha_{i_{1}}+\cdots+\alpha_{i_{s}}$ is uniquely determined by $\beta$. Moreover, $\beta$ is not a term of any chain of roots of the form $-\alpha_{i_{1}},-\alpha_{i_{1}}$ $-\alpha_{i_{2}}, \cdots,-\alpha_{i_{1}}-\cdots-\alpha_{i_{u}}=\beta, 1 \leqq i_{j} \leqq r$.

Proof. The existence of such a chain is readily proved by induction on $L(\beta)$. If $L(\beta)=1$, then $\beta=\alpha_{i}$ for some $i$, and we have the result. If $L(\beta)>1$, then by (c) and (d), $\beta-\alpha_{i}=\gamma$ is a root of level $L(\beta)-1>0$ for some $\alpha_{i}$. We may assume the existence of a chain of roots $\alpha_{i_{1}}, \alpha_{i_{1}}+\alpha_{i_{2}}, \cdots, \alpha_{i_{1}}+\cdots$ $+\alpha_{i_{s}}=\gamma$. Thus we have the chain of roots $\alpha_{i_{1}}, \alpha_{i_{1}}+\alpha_{i_{2}}, \cdots, \alpha_{i_{1}}+\cdots+\alpha_{i_{\mathrm{g}}}$, $\alpha_{i_{1}}+\cdots+\alpha_{i_{s}}+\alpha_{i}=\beta$.

If in a second such chain, we have $\beta=\alpha_{j_{1}}+\cdots+\alpha_{j_{\Delta}}$, where each $\alpha_{i}$ occurs $t^{\prime}(\beta, i)$ times, then by $(\mathrm{g})$ above, we have

$$
A_{\beta, \alpha_{j}}=\sum_{i=1}^{r} t(\beta, i) A_{i j}=\sum_{i=1}^{r} t^{\prime}(\beta, i) A_{i j}, \quad 1 \leqq j \leqq r .
$$

By (f), it follows that $t^{\prime}(\beta, i)=t(\beta, i), 1 \leqq i \leqq r$.

From (b) and (d), $L(\beta)=s$. If $\beta=-\alpha_{i_{1}}-\cdots-\alpha_{i_{u}}$ is also a term of a "negative" chain, then from (b) and (d), $L(\beta)=-u$, a contradiction. Hence Lemma 1 is proved.

Since $-\beta$ is a root if and only if $\beta$ is a root, it follows by (e) and Lemma 1 that if $L(\beta)<0$, then there is a chain $-\alpha_{i_{1}},-\alpha_{i_{1}}-\alpha_{i_{2}}, \cdots,-\alpha_{i_{1}}-\cdots$ $-\alpha_{i_{s}}=\beta$ of roots, where the number of times $-\alpha_{i}$ occurs is unique and is $t(-\beta, i)$, and that $\beta$ is not a member of any "positive" chain. In this case, we set $t(\beta, i)=-t(-\beta, i) \leqq 0,1 \leqq i \leqq r$. Then for every $\operatorname{root} \beta$, there is a uniquely determined expression $\beta=\sum_{i=1}^{r} t(\beta, i) \alpha_{i}$, where the $t(\beta, i)$ satisfy the above conditions. We call this representation the canonical representation of $\beta$ in terms of the fundamental system $\alpha_{1}, \cdots, \alpha_{r}$.

Lemma 2. The set of all $r$-tuples of natural numbers $(t(\beta, 1), \cdots, t(\beta, r))$, formed for all roots $\beta$, depends only on the matrix $\left(A_{i j}\right)$, and is independent of the base field.

Proof. Let $\mathfrak{M}$ be a second simple Lie algebra of classical type relative to a standard Cartan subalgebra $\Re$ over a base field $\&$ (of characteristic different from 2,3 ) and let $\alpha_{1}^{\prime}, \ldots, \alpha_{r}^{\prime}$ be a fundamental system of roots relative to $\Omega$ such that $A_{\alpha_{i}^{\prime}, \alpha_{j}^{\prime}}=A_{\alpha_{i}, \alpha_{j}}$ for all $i, j$. Let $\beta$ be a root of $\&$ relative to $\mathfrak{S}, \beta$ $=\sum_{i} t(\beta, i) \alpha_{i}$ its canonical representation. By symmetry it is enough to show that there is a root $\beta^{\prime}$ of $\mathfrak{M}$ relative to $\Omega$ with the canonical representa- 
tion $\beta^{\prime}=\sum_{i} t(\beta, i) \alpha_{i}^{\prime}$. Moreover, it is enough to treat only the case $L(\beta)>0$ (by the remarks immediately preceding this lemma).

Again we proceed by induction on $L(\beta)$. If $L(\beta)=1$, then $\beta=\alpha_{i}$ for some $i, t(\beta, j)=\delta_{i j}$, and $\alpha_{i}^{\prime}=\sum_{j} t(\beta, j) \alpha_{j}^{\prime}$ is the canonical representation of the root $\alpha_{i}^{\prime}$ of $\mathfrak{M}$. Now let $L(\beta)>1$, and assume the result for all roots of $\mathfrak{R}$ of positive level less than $L(\beta)$. By (C), $\beta-\alpha_{i}$ is a root for some $\alpha_{i}$, and $\beta \neq \alpha_{i}$ since $L(\beta)>1$. Let $m$ be the smallest positive integer such that $\beta-(m+1) \alpha_{i}$ is not a root of $\&$. Then $1 \leqq m \leqq 3$, the second inequality by the fact that $\beta, \beta-\alpha_{i}, \beta-2 \alpha_{i}, \beta-3 \alpha_{i}, \beta-4 \alpha_{i}$ cannot all be roots ((v) and (xvii) of (C)). Now $L\left(\beta-m \alpha_{i}\right)=L(\beta)-m<L(\beta)$, and $L\left(\beta-m \alpha_{i}\right)>0$ since if $L\left(\beta-m \alpha_{i}\right) \leqq 0$, then either $L\left(\beta-2 \alpha_{i}\right)=0$ or $L\left(\beta-3 \alpha_{i}\right)=0$, and $\beta=2 \alpha_{i}$ or $\beta=3 \alpha_{i}$. By (xvi) of (C), neither of these can be the case, and $L\left(\beta-m \alpha_{i}\right)>0$. If $\alpha=\beta-m \alpha_{i}$, we have $t(\alpha, j)=t(\beta, j)-m \delta_{i j}$, since if $\alpha_{i_{1}}, \cdots, \alpha_{i_{1}}+\cdots+\alpha_{i_{n}}=\alpha$ is a chain for $\alpha$, the chain $\alpha_{i_{1}}, \cdots, \alpha_{i_{1}}+\cdots+\alpha_{i_{n}}, \alpha_{i_{1}}+\cdots+\alpha_{i_{n}}+\alpha_{i}, \cdots, \alpha_{i_{1}}+\cdots$ $+\alpha_{i_{n}}+m \alpha_{i}=\beta$ contains all $\alpha_{j}$ for $j \neq i$ precisely $t(\alpha, j)$ times and contains $\alpha_{i}$ exactly $t(\alpha, i)+m$ times. By assumption, there is a root $\alpha^{\prime}$ of $\mathfrak{M}$ with canonical representation $\alpha^{\prime}=\sum_{j} t(\alpha, j) \alpha_{j}^{\prime}$. But then by (g) we have

$$
A_{\alpha^{\prime}, \alpha_{i}^{\prime}}=\sum_{j} t(\alpha, j) A_{\alpha_{j}^{\prime}, \alpha_{i}}^{\prime}=\sum_{j} t(\alpha, j) A_{j i}=A_{\alpha, \alpha_{i}} \leqq-m .
$$

Thus $\alpha^{\prime}, \alpha^{\prime}+\alpha_{i}^{\prime}, \cdots, \alpha^{\prime}+m \alpha_{i}^{\prime}=\beta^{\prime}$ are roots of $\mathfrak{M}$, and $t\left(\beta^{\prime}, j\right)=t\left(\alpha^{\prime}, j\right)$ $=t(\alpha, j)=t(\beta, j)$ if $j \neq i, t\left(\beta^{\prime}, i\right)=t\left(\alpha^{\prime}, i\right)+m=t(\alpha, i)+m=t(\beta, i)$. Thus $\beta^{\prime}$ has the canonical representation $\beta^{\prime}=\sum_{j} t(\beta, j) \alpha_{j}^{\prime}$, and Lemma 2 is proved by induction. (It will be noted that Lemma 2 is essentially proved in [14] by explicit computation of the roots.)

Lemma 3. Let $\mathfrak{R}$ and $\mathfrak{M}$ be simple Lie algebras of classical type over fields $\mathfrak{F}, \mathbb{E}$, respectively, with standard Cartan subalgebras $\mathfrak{S}, \Omega$ respectively. Let $\alpha_{1}, \cdots, \alpha_{r}$ be a fundamental system of roots for $\mathfrak{R}$ relative to $\mathfrak{S}, \alpha_{1}^{\prime}, \cdots, \alpha_{r}^{\prime}$ a fundamental system of roots for $\mathfrak{M}$ relative to $\Omega$, and suppose that $A_{\alpha_{i}, \alpha_{j}}=A_{\alpha_{i}^{\prime}, \alpha_{j}^{\prime}}$ $=A_{i j}, 1 \leqq i, j \leqq r$. Then there is a 1-1 mapping $\beta \rightarrow \beta^{\prime}$ of the set of roots of $\mathbb{R}$ relative to $\mathfrak{S}$ onto the set of roots of $\mathfrak{M}$ relative to $\Omega$ such that:

(1) $\alpha_{i} \rightarrow \alpha_{i}^{\prime}, 1 \leqq i \leqq r$;

(2) if $\beta \rightarrow \beta^{\prime}$, then $(-\beta) \rightarrow-\beta^{\prime}$;

(3) if $\alpha, \beta$, and $\alpha+\beta$ are roots, then $(\alpha+\beta)^{\prime}=\alpha^{\prime}+\beta^{\prime}$; if $\alpha$ and $\beta$ are roots, and if $\alpha^{\prime}+\beta^{\prime}$ is a root, then $(\alpha+\beta)^{\prime}=\alpha^{\prime}+\beta^{\prime}$;

(4) $A_{\alpha, \beta}=A_{\alpha^{\prime}, \beta^{\prime}}$ for all $\alpha, \beta$;

(5) $\left(\beta S_{i}\right)^{\prime}=\beta^{\prime} S_{\alpha_{i}}$ for all roots $\beta$ and all $i, 1 \leqq i \leqq r$.

Proof. Let $\beta$ be a root of $\mathcal{Q}$ relative to $\mathfrak{T}, \beta=\sum_{i} t(\beta, i) \alpha_{i}$ its canonical representation. We have seen in Lemma 2 that $\beta \rightarrow \beta^{\prime}=\sum_{i} t(\beta, i) \alpha_{i}^{\prime}$ defines a 1-1 mapping of the set of roots of $\mathbb{R}$ relative to $\mathfrak{S}$ onto the set of roots of $\mathfrak{M}$ relative to $\Omega$. Moreover, $\beta^{\prime}=\sum_{i} t(\beta, i) \alpha_{i}^{\prime}$ is the canonical representation of $\beta^{\prime}$ relative to $\alpha_{1}^{\prime}, \cdots, \alpha_{r}^{\prime}$, and $\alpha_{i} \rightarrow \alpha_{i}^{\prime}, 1 \leqq i \leqq r$. Since $-\beta=\sum t(-\beta, i) \alpha_{i}$ $\rightarrow \sum t(-\beta, i) \alpha_{i}^{\prime}=-\sum t(\beta, i) \alpha_{i}^{\prime}=-\beta^{\prime}$, (2) is satisfied. If $\alpha, \beta$, and $\alpha+\beta=\gamma$ 
are roots of $\mathfrak{R}$, let $\gamma=\sum t(\gamma, i) \alpha_{i}$ be the canonical representation of $\gamma$. Then $\gamma^{\prime}=\sum t(\gamma, i) \alpha_{i}^{\prime}$ is the canonical representation of a root of $\mathfrak{M}$, as are $\alpha^{\prime}$ $=\sum t(\alpha, i) \alpha_{i}^{\prime}$ and $\beta^{\prime}=\sum t(\beta, i) \alpha_{i}^{\prime}$. Since $\gamma=\alpha+\beta$, we see by $(\mathrm{g})$ that

$$
A_{\gamma, \alpha_{j}}=\sum_{i} t(\gamma, i) A_{i j}=A_{\alpha, \alpha_{j}}+A_{\beta, \alpha_{j}}=\sum_{i}(t(\alpha, i)+(\beta, i)) A_{i j},
$$

$1 \leqq j \leqq r$. Hence $t(\gamma, i)=t(\alpha, i)+t(\beta, i), 1 \leqq i \leqq r$, by (f), and $\alpha^{\prime}+\beta^{\prime}=\gamma^{\prime}$ $=(\alpha+\beta)^{\prime}$. A completely analogous argument establishes the converse part of (3).

The result (4) follows from (3): if $\alpha-q \beta, \cdots, \alpha-\beta, \alpha, \alpha+\beta, \cdots, \alpha+m \beta$ are roots, and $\alpha-(q+1) \beta$ and $\alpha+(m+1) \beta$ are not roots, where $m$ and $q$ are non-negative integers, the corresponding assertion holds for the sequence $\alpha^{\prime}-q \beta^{\prime}, \cdots, \alpha^{\prime}-\beta^{\prime}, \alpha^{\prime}, \alpha^{\prime}+\beta^{\prime}, \cdots, \alpha^{\prime}+m \beta^{\prime}$. Thus $A_{\alpha, \beta}=q-m=A_{\alpha^{\prime}, \beta^{\prime}}$. Finally, $\beta S_{i}=\beta-A_{\beta, \alpha_{i}} \alpha_{i}$ is a root; it will be enough to prove (5) in case $L(\beta)>0$. First let $\beta=\alpha_{i}$; then $\beta S_{i}=-\alpha_{i}$ and $\left(\beta S_{i}\right)^{\prime}=-\alpha_{i}^{\prime}=\alpha_{i}^{\prime} S_{\alpha_{i}^{\prime}}=\beta^{\prime} S_{\alpha_{i}}$. If $\beta \neq \alpha_{i}$, let $A_{\beta, \alpha_{i}} \geqq 0$; then $L\left(\beta S_{i}\right)>0$ since $\beta$ is not a multiple of $\alpha_{i}$. Now $\beta S_{i}, \beta S_{i}+\alpha_{i}, \cdots, \beta S_{i}+A_{\beta, \alpha_{i}} \alpha_{i}=\beta$ are all roots, yielding $t(\beta, j)=t\left(\beta S_{i}, j\right)$ $+A_{\beta, \alpha_{i}} \delta_{i j}, t\left(\beta S_{i}, j\right)=t(\beta, j)-A_{\beta, \alpha_{i}} \delta_{i j}=t\left(\beta^{\prime}, j\right)-A_{\beta^{\prime}, \alpha_{i}^{\prime}} \delta_{i j}=t\left(\beta^{\prime} S_{\alpha_{i}}^{\prime}, j\right)$ by the same reasoning, $1 \leqq i, j \leqq r$. Therefore $\left(\beta S_{i}\right)^{\prime}=\beta^{\prime} S_{\alpha_{i}}, 1 \leqq i \leqq r$. An analogous argument proves $\left(\beta S_{i}\right)^{\prime}=\beta^{\prime} S_{\alpha_{i}^{\prime}}$ if $A_{\beta, \alpha_{i}}<0$. Thus Lemma 3 is proved.

Lemma 4. Let the notations be as in Lemma 3. Then if $\beta_{1}, \cdots, \beta_{r}$ is any fundamental system of roots for $\&$ relative to $\mathfrak{S}$, and if $\beta_{1}^{\prime}, \cdots, \beta_{r}^{\prime}$ are their images under the mapping $\beta \rightarrow \beta^{\prime}$, then $\beta_{1}^{\prime}, \cdots, \beta_{r}^{\prime}$ is a fundamental system of roots for $\mathfrak{M}$ relative to $\Re$, and $A_{\beta_{i}, \beta_{j}}=A_{\beta_{i}, \beta_{j}}$ for all $i, j$. Conversely, if $\beta_{1}^{\prime}, \cdots, \beta_{r}^{\prime}$ is a fundamental system relative to $\Omega$, then the preimages $\beta_{1}, \cdots, \beta_{r}$ constitutc a fundamental system relative to $\mathfrak{S}$, and $A_{\beta_{i}, \beta_{j}}=A_{\beta_{i}, \beta_{j}}$ for all $i, j$.

Proof. By the symmetry with its inverse of the mapping $\beta \rightarrow \beta^{\prime}$, and by (4) of Lemma 3 , it is enough to show that if $\beta_{1}, \cdots, \beta_{r}$ is a fundamental system, then so is $\beta_{1}^{\prime}, \cdots, \beta_{r}^{\prime}$. Now if $\beta_{1}, \cdots, \beta_{r}$ is fundamental, then by Lemma 1 every nonzero root $\beta$ relative to $\mathfrak{S}$ occurs either in a chain of roots $\beta_{i_{1}}, \beta_{i_{1}}+\beta_{i_{2}}, \cdots, \beta_{i_{1}}+\cdots+\beta_{i_{s}}=\beta$ or in a chain $-\beta_{i_{1}},-\beta_{i_{1}}-\beta_{i_{2}}, \cdots$, $-\beta_{i_{1}}-\cdots-\beta_{i_{\mathrm{s}}}=\beta, 1 \leqq i_{j} \leqq r$. Since the images $\beta^{\prime}$ exhaust all roots relative to $\Omega$, it follows from (2) and (3) of Lemma 3 that every nonzero root $\beta^{\prime}$ relative to $\Re$ occurs either in a chain of roots $\beta_{i_{1}}^{\prime}, \beta_{i_{1}}^{\prime}+\beta_{i_{2}}^{\prime}, \cdots, \beta_{i_{1}}^{\prime}+\cdots+\beta_{i_{s}}^{\prime}$ $=\beta^{\prime}$ or in a chain $-\beta_{i_{1}}^{\prime}, \quad-\beta_{i_{1}}^{\prime}-\beta_{i_{2}}^{\prime}, \cdots,-\beta_{i_{1}}^{\prime}-\cdots-\beta_{i_{s}}=\beta^{\prime}$. Thus $\beta_{1}^{\prime}, \cdots, \beta_{r}^{\prime}$ spans $\Omega^{*}$, since the roots span $\Omega^{*}$. Next, $\beta_{1}, \cdots, \beta_{r}$ is indecomposable and $\left(A_{\beta_{i}, \beta_{j}}\right)$ is nonsingular; since $A_{\beta_{i}, \beta_{j}}=A_{\beta_{i}, \beta_{j}}$, the same properties hold for $\beta_{1}^{\prime}, \cdots, \beta_{r}^{\prime}$. If $\beta_{i}^{\prime}-\beta_{j}^{\prime}$ were a root for some $i \neq j$, then the preimage $\beta_{i}-\beta_{j}$ would be a root, contrary to assumption. Finally, let $\delta$ be that unique root relative to $\mathfrak{S}$ such that no $\delta-\beta_{i}$ is a root. Then no $\delta^{\prime}-\beta_{i}^{\prime}$, $1 \leqq i \leqq r$, is a root relative to $\Omega$, and $\delta^{\prime}$ is a root relative to $\Omega$ with the same property of minimality. If $\gamma^{\prime}$ were a second root relative to $R$ such that no $\gamma^{\prime}-\beta_{i}^{\prime}$ is a root, then no $\gamma-\beta_{i}$ would be a root, where $\gamma$ is the preimage of 
$\gamma^{\prime}$. By the uniqueness of $\delta, \gamma=\delta$, and $\gamma^{\prime}=\delta^{\prime}$. Thus the proof of Lemma 4 is complete.

Lemma 5. With the notations of Lemmas 2 and 3, the mappings $\beta \rightarrow \beta^{\prime}$ and $S_{\alpha_{i}} \rightarrow S_{\alpha_{i}}$ define an isomorphism of the Weyl group $\mathfrak{B}$ relative to $\mathfrak{S}$ (generated by the $S_{\alpha_{i}}$ ), regarded as a transformation group on the roots relative to $\mathfrak{S}$, onto the Weyl group $\mathfrak{W}^{\prime}$ relative to $\Re$ (generated by the $S_{\alpha_{i}}$ ), regarded as a transformation group on the roots relative to $\Re$. That is, the mapping $S_{\alpha_{i}} \rightarrow S_{\alpha_{i}}$ can be uniquely extended to an isomorphism $\tau$ of $\mathfrak{W}$ onto $\mathfrak{W}^{\prime}$ such that for every root $\beta$ relative to $\mathfrak{S}$ and for every $S \in \mathfrak{B},(\beta S)^{\prime}=\beta^{\prime} S^{\top}$.

Proof. From (5) of Lemma 3, $\left(\beta S_{\alpha_{i}}\right)^{\prime}=\beta^{\prime} S_{\alpha_{i}}$ for all roots $\beta$. It follows that, in general, $\left(\beta S_{\alpha_{i_{1}}} \cdots S_{\alpha_{i_{k}}}\right)^{\prime}=\beta^{\prime} S_{\alpha_{i_{1}}^{\prime}}^{\prime} \cdots S_{\alpha_{i_{k}}}$ for all $\beta$ and for $1 \leqq i_{j} \leqq r$. Thus if $S_{\alpha_{i_{1}}} \cdots S_{\alpha_{i_{k}}}=S_{\alpha_{j_{1}}} \cdots S_{\alpha_{j_{m}}}$ then $\beta^{\prime} S_{\alpha_{i_{1}}^{\prime}}^{\prime} \cdots S_{\alpha i_{k}}=\beta^{\prime} S_{\alpha_{j_{1}}^{\prime}}^{\prime} \cdots S_{\alpha_{j_{m}}^{\prime}}$ for all roots $\beta^{\prime}$ relative to $\AA$, so that the mapping $\tau:\left(S_{\alpha_{i_{1}}{ }^{\prime}} \cdots S_{\alpha_{i_{k}}}\right)^{r}=S_{\alpha_{i_{1}}{ }^{\prime}} \cdots S_{\alpha^{\prime}{ }_{i_{k}}}$ defines a homomorphism of $\mathfrak{W}$ onto $\mathfrak{W}^{\prime}$. By symmetry, the mapping $\omega:\left(S_{\alpha^{\gamma_{i_{1}}}} \cdots S_{\alpha_{i_{k}}}\right)^{\omega}=S_{\alpha_{i_{1}}} \cdots S_{\alpha_{i_{k}}}$ defines a homomorphism of $\mathfrak{W}^{\prime}$ onto $\mathfrak{W}$ such that $\tau \omega$ is the identity on $\mathfrak{W}, \omega \tau$ the identity on $\mathfrak{W}^{\prime}$. Hence $\tau$ is an isomorphism with inverse $\omega$, and $\tau$ is the only possible homomorphic extension to $\mathfrak{W}$ of the mapping $S_{\alpha_{i}} \rightarrow S_{\alpha_{i}}$. By the above, we have $(\beta S)^{\prime}=\beta^{\prime} S^{\tau}$ for all roots $\beta$ relative to $\mathfrak{S}$ and for all $S \in \mathfrak{B}$. This completes the proof.

THEOREM 2. Let $\mathfrak{R}$ be a simple Lie algebra of classical type over a field $\mathfrak{F}$ of characteristic not 2 or 3 . Let $\mathfrak{S}$ be a standard Cartan subalgebra of $\mathfrak{Z}$ and $\alpha_{1}, \cdots, \alpha_{r}$ a fundamental system of roots relative to $\mathfrak{S}$. Let $\mathfrak{W}$ be the group generated by the $S_{\alpha_{i}}$. Then $\mathfrak{B}$ is independent of the particular fundamental system $\alpha_{1}, \cdots, \alpha_{r}$ chosen, and if $\beta_{1}, \cdots, \beta_{s}$ is a second fundamental system relative to $\mathfrak{S}$, then $s=r$ and there is a unique $S \in \mathfrak{W}$ such that $\beta_{1}, \cdots, \beta_{r}$ is a permutation of $\alpha_{1} S, \cdots, \alpha_{r} S$. For every $S \in \mathfrak{B}, \alpha_{1} S, \cdots, \alpha_{r} S$ is a fundamental system relative to $\mathfrak{H}$.

Proof. First let $\&$ be a simple Lie algebra over an algebraically closed field of characteristic zero. It should first be noted that our notion of fundamental system of roots then coincides with the traditional notion of a fundamental system, namely, a linearly independent set of roots such that every root is a linear combination of this set with nonpositive integral coefficients or a linear combination of this set with non-negative integral coefficients. For by Lemma 24 of (C), a fundamental system in the sense of (C) is a basis for $\mathfrak{S}^{*}$ if the base field is of characteristic zero, and in this case every root is either a nonnegative or nonpositive integral combination of the system by Lemma 1 above. Conversely, if $\mathfrak{F}$ is algebraically closed of characteristic zero and if $\alpha_{1}, \cdots, \alpha_{r}$ is a fundamental system for $\mathfrak{l}$ in the traditional sense, then the matrix $\left(\alpha_{i}\left(h_{j}\right)\right)=\left(A_{i j} \cdot 1\right)$ is known to be indecomposable and nonsingular (see $[5 ; 6 ; 16]$ ), and $\alpha_{1}, \cdots, \alpha_{r}$ spans $\mathfrak{S}^{*}$ by definition. Since $\mathfrak{F}$ is of characteristic zero, and since there are only finitely many roots relative to $\mathfrak{S}$, 
no string of roots $\beta, \beta-\alpha_{i_{1}}, \cdots, \beta-\alpha_{i_{1}}-\alpha_{i_{2}}-\cdots-\alpha_{i_{2}}, \cdots$ can continue indefinitely. Hence there must be a root $\delta$ such that no $\delta-\alpha_{i}$ is a root. But then $\delta$ is the highest weight of the adjoint representation of $\&$ on itself relative to the fundamental system $-\alpha_{1},-\alpha_{2}, \cdots,-\alpha_{r}$ (in the traditional sense). Since this representation is irreducible by the simplicity of $\mathfrak{R}$, no other weight (root, in this case) $\gamma$ can have the property that no $\gamma-\alpha_{i}$ is a root (see $[1 ; 16 ; 18])$. Thus the two senses of fundamental system are equivalent for $\mathbb{R}$.

Let $\Lambda$ be a linear function on $\mathfrak{S}$ such that $\Lambda\left(h_{i}\right)=m_{i} \cdot 1$, where the $m_{i}$, $1 \leqq i \leqq r$, are distinct positive integers. Then there is an irreducible representation of $\&$ with highest weight $\Lambda$ (relative to $\alpha_{1}, \cdots, \alpha_{r}$; see $[1 ; 8 ; 16 ; 18]$ for the results on representations used in this paragraph). Since each $S \in \mathfrak{W}$ is linear, nonsingular, and permutes the roots, it follows that $\alpha_{1} S, \cdots, \alpha_{r} S$ is a fundamental system for each $S \in \mathfrak{B}$. Conversely, if $\beta_{1}, \cdots, \beta_{s}$ is a fundamental system, then $s=\operatorname{dim} \mathfrak{S}=r$, and our irreducible representation of $\mathbb{R}$ has a unique highest weight $\mathrm{M}$ relative to $\beta_{1}, \cdots, \beta_{r}$. Then $\mathrm{M}$ is an extreme weight in the sense of $\$ 4$ of [1], from which it follows that there is an $S \in \mathfrak{B}$ such that $\mathrm{M} S=\Lambda$. Now $\mathrm{M} S=\Lambda$ is the highest weight relative to $\beta_{1} S, \cdots, \beta_{r} S$. If $\gamma=\beta_{i} S$ is not a positive root (relative to $\alpha_{1}, \cdots, \alpha_{r}$ ) for some $i$, then $\gamma=\sum n_{j} \alpha_{j}, n_{j}$ integers $\leqq 0$, some $n_{j}<0$. Let $h \in\left[L_{-\gamma} L_{\gamma}\right], \gamma(h)=2$. Then $h=\sum \rho_{j} h_{j}, \rho_{j} \in \mathfrak{F}$. From $\alpha_{k}(h)=2\left(\alpha_{k}, \gamma\right)(\gamma, \gamma)^{-1}$, where the scalar product is that induced on $\mathfrak{S}^{*}$ by the Killing form on $\mathfrak{E}$, we obtain

$$
\begin{aligned}
\alpha_{k}(h) & =\sum_{j} \rho_{j} A_{k j}=2(\gamma, \gamma)^{-1} \sum_{j} n_{j}\left(\alpha_{k}, \alpha_{j}\right) \\
& =(\gamma, \gamma)^{-1} \sum_{j} n_{j}\left(\alpha_{j}, \alpha_{j}\right) A_{k j},
\end{aligned}
$$

By the nonsingularity of the matrix $\left(A_{k j}\right), \rho_{j}=(\gamma, \gamma)^{-1}\left(\alpha_{j}, \alpha_{j}\right) n_{j}, 1 \leqq j \leqq r$, and for all roots $\alpha,(\alpha, \alpha)$ is known to be a positive rational number (identifying the rationals under the only possible isomorphism with the prime field of $\mathfrak{F}$ ) [16]. From $0 \leqq 2(\Lambda, \gamma)(\gamma, \gamma)^{-1}=\Lambda(h)=\sum_{j} \rho_{j} m_{j}$, we have $\sum_{j} m_{j} n_{j}\left(\alpha_{j}, \alpha_{j}\right) \geqq 0$. But all $m_{j}>0$ and all $\left(\alpha_{j}, \alpha_{j}\right)>0$, so that $\sum_{j} m_{j} n_{j}\left(\alpha_{j}, \alpha_{j}\right)<0$, a contradiction. It follows that each $\beta_{i} S$ is a positive root with respect to $\alpha_{1}, \cdots, \alpha_{r}$, and conversely each $\alpha_{i}$ is positive with respect to $\beta_{1} S, \cdots, \beta_{r} S$. An elementary argument shows that this is impossible unless $\beta_{1} S, \cdots, \beta_{r} S$ is a permutation of $\alpha_{1}, \cdots, \alpha_{r}$. Thus every fundamental system is an image of $\alpha_{1}, \cdots, \alpha_{r}$ under $\mathfrak{B}$. If $S \in \mathfrak{B}$ permutes $\alpha_{1}, \cdots, \alpha_{r}$, then $\Lambda S=\Lambda$ is the highest weight relative to $\alpha_{1} S, \cdots, \alpha_{r} S$ and if $\alpha_{i} S=\alpha_{i \phi}, \phi$ a permutation of $(1, \cdots, r)$, we have $\Lambda S\left(h_{i \phi}\right)=\Lambda\left(h_{i}\right)$, all $i$, or $\Lambda\left(h_{i \phi}\right)=\Lambda\left(h_{i}\right)$, all $i$. Since all $\Lambda\left(h_{i}\right)$ are distinct, $i \phi=i$ for all $i$, and $S$ is the identity. The uniqueness assertion of the theorem now follows in this case.

(The referee has pointed out that in case $\mathfrak{F}$ is the complex field, a proof of the assertions of the theorem which is independent of some of the results essential to the argument above has been given by Satake [20]. For the next part of the proof one can take the field $\&$ to be the complex field and can ap- 
ply Satake's result instead of the above.)

Now let $\mathfrak{R}, \mathfrak{F}, \mathfrak{S}, \alpha_{1}, \cdots, \alpha_{r}, \mathfrak{W}$, and $\beta_{1}, \cdots, \beta$, be as in the statement of the theorem. Then there is a simple Lie algebra $\mathfrak{M}$ over an algebraically closed field $\&$ of characteristic zero with standard Cartan subalgebra $\Omega$ and fundamental system of roots $\alpha_{1}^{\prime}, \cdots, \alpha_{r}^{\prime}$ relative to $\Omega$ such that $A_{\alpha_{i}^{\prime}, \alpha_{j}^{\prime}}$ $=A_{\alpha_{i}, \alpha_{j}}, 1 \leqq i, j \leqq r$ (see Theorem 6 of $(\mathrm{C})$ and, for example, $\left.[5 ; 8 ; 19]\right)$. Let $\mathfrak{W}^{\prime}$ be the Weyl group of $\mathfrak{M}$ relative to $\Omega$ (generated by the $S_{\alpha_{i}}^{\prime}$ ). Let the correspondence $\beta \rightarrow \beta^{\prime}$ and the isomorphism $\tau$ of $\mathfrak{W}$ onto $\mathfrak{W}^{\prime}$ be as in Lemmas 3-5.

If $\beta$ is any root relative to $\mathfrak{E}(\beta \neq 0)$ and $\gamma$ is any other root, then $\gamma S_{\beta}$ $=\gamma-A_{\gamma, \beta} \beta$. We wish to show $S_{\beta} \in \mathfrak{W}$. But $\gamma^{\prime} S_{\beta^{\prime}}=\gamma^{\prime}-A_{\gamma^{\prime}, \beta} \beta^{\prime}=\gamma^{\prime}-A_{\gamma, \beta} \beta^{\prime}$ and $S_{\beta}, \in \mathfrak{W}^{\prime}$ by our earlier remarks based on [16]. If $T=S_{\beta}^{r^{-1}}$, then $(\gamma T)^{\prime}$ $=\gamma^{\prime} T^{\gamma}=\gamma^{\prime} S_{\beta^{\prime}}=\gamma^{\prime}-A_{\gamma, \beta} \beta^{\prime}$, so that $\gamma T=\gamma-A_{\gamma, \beta} \beta=\gamma S_{\beta}$ for all roots $\gamma$. Thus $S_{\beta}=T \in \mathfrak{B}$, and $S_{\beta}^{\tau}=S_{\beta^{\prime}}$. It follows that $\mathfrak{B}$ is independent of the fundamental system $\alpha_{1}, \cdots, \alpha_{r}$ used in its generation. If $\beta_{1}, \cdots, \beta_{s}$ is a second fundamental system relative to $\mathfrak{E}$, then by Lemma $4, \beta_{1}^{\prime}, \ldots, \beta_{s}^{\prime}$ is a fundamental system relative to $\Re$. Hence $r=s$ by the results at characteristic zero, and there is a unique $U \in \mathfrak{W}^{\prime}$ such that $\beta_{1}^{\prime}, \ldots, \beta_{r}^{\prime}$ is a permutation of $\alpha_{1}^{\prime} U, \cdots, \alpha_{r}^{\prime} U$. Let $S=U^{r^{-1}} \in \mathfrak{B}$. Then $\left(\alpha_{i} S\right)^{\prime}=\alpha_{i}^{\prime} S^{r}=\alpha_{i}^{\prime} U, 1 \leqq i \leqq r$, and $\left(\alpha_{1} S\right)^{\prime}, \cdots,\left(\alpha_{r} S\right)^{\prime}$ is a permutation of $\beta_{1}^{\prime}, \cdots, \beta_{r}^{\prime}$. Hence $\alpha_{1} S, \cdots, \alpha_{r} S$ is a permutation of $\beta_{1}, \cdots, \beta_{r}$. If $\alpha_{1} T, \cdots, \alpha_{r} T$ is also a permutation of $\beta_{1}, \cdots, \beta_{r}$, where $T \in \mathfrak{W}$, then $\left(\alpha_{1} T\right)^{\prime}, \cdots,\left(\alpha_{r} T\right)^{\prime}$ is a permutation of $\beta_{1}^{\prime}, \ldots, \beta_{r}^{\prime}$, i.e., $\alpha_{1}^{\prime} T^{\tau}, \cdots, \alpha_{r}^{\prime} T^{\tau}$ is a permutation of $\beta_{1}^{\prime}, \cdots, \beta_{r}^{\prime}$. Hence $T^{\tau}=U=S^{\tau}$, and $T=S$. Finally if $S \in \mathfrak{W}$, then $S^{\tau} \in \mathfrak{W}^{\prime}$, and $\alpha_{1}^{\prime} S^{\tau}, \cdots, \alpha_{r}^{\prime} S^{\tau}$ is a fundamental system relative to $\Omega$. That is, $\left(\alpha_{1} S\right)^{\prime}, \ldots,\left(\alpha_{r} S\right)^{\prime}$ is a fundamental system relative to $\Omega$. By Lemma $4, \alpha_{1} S, \ldots, \alpha_{r} S$ is a fundamental system relative to $\mathfrak{E}$, and the proof of Theorem 2 is complete.

III. Automorphisms mapping a Cartan subalgebra into itself. Let $\&$ be simple, $\mathfrak{S}$ a standard Cartan subalgebra, $\alpha_{1}, \cdots, \alpha_{r}$ a fundamental system of roots relative to $\mathfrak{S}$. Let $h_{i}$ be as before, i.e., $h_{i} \in\left[\mathcal{R}_{\alpha_{i}} \Omega_{-\alpha_{i}}\right], \alpha_{i}\left(h_{i}\right)=2$, $1 \leqq i \leqq r$, and let $e_{\alpha_{i}} \in \mathfrak{R}_{\alpha_{i}}, e_{-\alpha_{i}} \in \mathfrak{R}_{-\alpha_{i}}$, with $\left[e_{-\alpha_{i}} e_{\alpha_{i}}\right]=h_{i}$. Let $0 \neq e_{\beta} \in \mathfrak{R}_{\beta}$ for each of the remaining roots $\beta$. Then the $h_{i}$ span $\mathfrak{S}$, and the $h_{i}$ together with all the $e_{\alpha}$ span $\&$ ((C), (xii) and Lemma 23). Also if $\alpha \neq-\gamma$, we have $\left[e_{\alpha} e_{\gamma}\right]=N_{\alpha, \gamma} e_{\alpha+\gamma}$, where $N_{\alpha, \gamma} \in \tilde{\mho}, N_{\alpha, \gamma} \neq 0$ if and only if $\alpha+\gamma$ is a root ((C), (xii) and (xiii)). Let $\lambda \neq 0$ be an element of $\mathfrak{F}, D_{i}=\operatorname{ad}\left(e_{\alpha_{i}}\right)$. We compute the effect of the automorphism $A_{i}(\lambda)=\exp \left(\lambda D_{i}\right)$ on $\mathfrak{S}$.

Now $\mathfrak{S}$ has a basis consisting of $h_{i}$ and a set of vectors $h$ such that $\alpha_{i}(h)=0$. For the latter, we have $h D_{i}=\left[h e_{\alpha_{i}}\right]=-\alpha_{i}(h) e_{\alpha_{i}}=0$, so that $h A_{i}(\lambda)=h$ if $\alpha_{i}(h)=0$. Also, $h_{i} D_{i}=\left[h_{i} e_{\alpha_{i}}\right]=-\alpha_{i}\left(h_{i}\right) e_{\alpha_{i}}=-2 e_{\alpha_{i}}$, and $h_{i} D_{i}^{2}=-2\left[e_{\alpha_{i}} e_{\alpha_{i}}\right]=0$. Hence $h_{i} A_{i}(\lambda)=h_{i}-2 \lambda e_{\alpha_{i}}$. Further, we note that $e_{\alpha_{i}} A_{i}(\lambda)=e_{\alpha_{i}}$, and that

$$
\begin{aligned}
e_{-\alpha_{i}} A_{i}(\lambda) & =e_{-\alpha_{i}}+\lambda\left[e_{-\alpha_{i}} e_{\alpha_{i}}\right]+\frac{\lambda^{2}}{2 !}\left[\left[e_{-\alpha_{i}} e_{\alpha_{i}}\right] e_{\alpha_{i}}\right] \\
& =e_{-\alpha_{i}}+\lambda h_{i}-\lambda^{2} e_{\alpha_{i}} .
\end{aligned}
$$


Next let $B_{i}(\mu)=\exp \left(\mu \operatorname{ad}\left(e_{-\alpha_{i}}\right)\right), \mu \in \mathfrak{F}$. Then if $\alpha_{i}(h)=0, h A_{i}(\lambda) B_{i}(\mu)=h$ as before. Also

$$
\begin{aligned}
h_{i} A_{i}(\lambda) B_{i}(\mu) & =\left(h_{i}-2 \lambda e_{\alpha_{i}}\right) B_{i}(\mu) \\
& =h_{i} B_{i}(\mu)-2 \lambda e_{\alpha_{i}} B_{i}(\mu) \\
& =h_{i}+2 \mu e_{-\alpha_{i}}-2 \lambda e_{\alpha_{i}}+2 \lambda \mu h_{i}+2 \lambda \mu^{2} e_{-\alpha_{i}} \\
& =2 \mu(1+\lambda \mu) e_{-\alpha_{i}}+(1+2 \lambda \mu) h_{i}-2 \lambda e_{\alpha_{i}} .
\end{aligned}
$$

In particular, $h_{i} A_{i}(\lambda) B_{i}\left(-\lambda^{-1}\right)=-h_{i}-2 \lambda e_{\alpha_{i}}$, and

$$
\begin{aligned}
h A_{i}(\lambda) B_{i}\left(-\lambda^{-1}\right) A_{i}(\lambda) & =h \text { if } \alpha_{i}(h)=0 ; \\
h_{i} A_{i}(\lambda) B_{i}\left(-\lambda^{-1}\right) A_{i}(\lambda) & =-h_{i}+2 \lambda e_{\alpha_{i}}-2 \lambda e_{\alpha_{i}}=-h_{i} .
\end{aligned}
$$

Thus $T(\lambda, i)=A_{i}(\lambda) B_{i}\left(-\lambda^{-1}\right) A_{i}(\lambda)$ is an invariant automorphism $\tau$ mapping $\mathfrak{S}$ onto itself, with $h \tau=h$ if $\alpha_{i}(h)=0$, and with $h_{i} \tau=-h_{i}$. If $\alpha$ is any root relative to $\mathfrak{S}, \alpha^{\tau}$ is defined by $\alpha^{\tau}(h \tau)=\alpha(h)$ for all $h \in \mathfrak{S}$. Since $\mathfrak{S} \tau=\mathfrak{S}, \alpha^{\tau}$ is also a root relative to $\mathfrak{S}$, with $\alpha^{\tau}(h)=\alpha(h)$ if $\alpha_{i}(h)=0$ and with $\alpha^{\tau}\left(h_{i}\right)=-\alpha\left(h_{i}\right)$. If $\alpha_{i}(h)=0$, we have $\left(\alpha S_{i}\right)(h)=\left(\alpha-\alpha\left(h_{i}\right) \alpha_{i}\right)(h)=\alpha(h)$, and we also have $\left(\alpha S_{i}\right)\left(h_{i}\right)$ $=\alpha\left(h_{i}\right)-\alpha\left(h_{i}\right) \alpha_{i}\left(h_{i}\right)=\alpha\left(h_{i}\right)-2 \alpha\left(h_{i}\right)=-\alpha\left(h_{i}\right)$. Thus $\alpha^{\tau}=\alpha S_{i}$ for all roots $\alpha$.

Now let $\sigma$ be any automorphism of $\mathfrak{R}$ mapping $\mathfrak{S}$ onto $\mathfrak{S}$. Then $\alpha_{1}^{\sigma}, \cdots, \alpha_{r}^{\sigma}$ is a fundamental system of roots relative to $\mathfrak{S} \sigma=\mathfrak{S}$ by Theorem 1. Therefore, by Theorem 2 , there is a unique $S \in \mathfrak{W}$ such that $\alpha_{1} S, \cdots, \alpha_{r} S$ is a permutation of $\alpha_{1}^{\sigma}, \cdots, \alpha_{r}^{\sigma}$. If $S=S_{i_{1}} \cdots S_{i_{k}}$, where $S_{i_{j}}=S_{\alpha_{i j}}, 1 \leqq i_{j} \leqq r$, and if $\lambda_{i_{j}}, 1 \leqq i_{j} \leqq r$, are nonzero elements of $\mathfrak{F}$, let $\tau=T\left(\lambda_{i_{1}}, i_{1}\right) \cdots T\left(\lambda_{i_{k}}, i_{k}\right)$. Then $\tau$ is an invariant automorphism of $\mathbb{\imath}$ mapping $\mathfrak{S}$ onto $\mathfrak{F}$, and $\alpha^{\tau}=\alpha S$ for all roots $\alpha$. Thus $\alpha_{1}^{\tau}, \cdots, \alpha_{r}^{\tau}$ are a permutation of $\alpha_{1}^{\sigma}, \cdots, \alpha_{r}^{\sigma}$, and $\alpha_{1}^{\sigma \tau^{-1}}, \cdots$, $\alpha_{r}^{\sigma^{-1}}$ is a fundamental system which is a permutation of $\alpha_{1}, \cdots, \alpha_{r}$. In addition, it follows from Theorem 1 that if $\beta_{i}=\alpha_{i}^{\sigma \tau^{-1}}, 1 \leqq i \leqq r$, then $A_{\beta_{i}, \beta_{j}}$ $=A_{\alpha_{i}, \alpha_{j}}, 1 \leqq i, j \leqq r$. Thus we have proved the following theorem:

TheOREm 3. Let $\mathfrak{\&}$ be a simple Lie algebra of classical type, $\mathfrak{S}$ a standard Cartan subalgebra, $\alpha_{1}, \cdots, \alpha_{r}$ a fundamental system of roots relative to $\mathfrak{S}$. Let $\sigma$ be an automorphism of $\mathfrak{R}$ such that $\mathfrak{S} \sigma=\mathfrak{S}$. Then there is an invariant automorphism $\tau$ of \& such that $\mathfrak{S} \tau=\mathfrak{F}$, and such that if $\beta_{i}=\alpha_{i}^{\sigma^{-1}}, 1 \leqq i \leqq r$, then $\beta_{1}, \cdots, \beta_{r}$ is a permutation of $\alpha_{1}, \cdots, \alpha_{r}$, with $A_{\beta_{i}, \beta_{j}}=A_{\alpha_{i}, \alpha_{j}}, 1 \leqq i, j \leqq r$.

Next let $\alpha_{1}, \cdots, \alpha_{r}$ and $\beta_{1}, \cdots, \beta_{r}$ be as in Theorem 3, with $\beta_{i}=\alpha_{i \phi}$, $\phi \in \Sigma_{r}$, the symmetric group on $r$ letters. Then $A_{i \phi, j \phi}=A_{i j}, 1 \leqq i, j \leqq r$. That is, the matrix with $A_{i \phi, j \phi}$ as the entry in the $i$ th row and $j$ th column is the matrix $\left(A_{i j}\right)$, or the Schläfli diagram of the (ordered) system $\alpha_{1 \phi}, \cdots, \alpha_{r \phi}$ is the same as that of $\alpha_{1}, \cdots, \alpha_{r}$. Thus $\alpha_{i} \rightarrow \alpha_{i \phi}$ effects a symmetry of this diagram (or of the corresponding matrix). (The diagrams by themselves do not display completely the matrices $\left(A_{i j}\right)$; in case a triple or double line is included, one must also consider the integers corresponding to its pair of endpoints.) Examination of the matrices of Table 2 of [6] (see note below), or of the 
diagrams of (C), shows immediately that $i=i \phi$ for all $i$ except in case $\alpha_{1}, \cdots, \alpha_{r}$ is of one of the types $A_{r}(r \geqq 2), D_{r}(r \geqq 4), E_{6}$. If $\alpha_{1}, \cdots, \alpha_{r}$ is of type $A_{r}$, and is labeled as in [6] or in (C), we have either $i \phi=i$ for all $i$, or $i \phi=r+1-i$ for all $i$. Similarly, if $\alpha_{1}, \cdots, \alpha_{r}$ is of type $D_{r}(r \geqq 5)$, then either $i \phi=i$ for all $i$, or $i \phi=i$ for all $i \leqq r-2$, and $(r-1) \phi=r, r \phi=r-1$. If $\alpha_{1}, \cdots, \alpha_{6}$ is of type $\mathrm{E}_{6}$, then either $i \phi=i$ for all $i$, or $6 \phi=6$ and $i \phi=6-i, 1 \leqq i \leqq 5$. If $\alpha_{1}, \cdots, \alpha_{4}$ is of type $D_{4}$, then $2 \phi=2$, and $1 \phi, 3 \phi, 4 \phi$ may be any one of the six possible permutations of $1,3,4$.

Note. In Table 2 of [6], we have $A_{i j}=g^{i j}$ for all $i, j$ in all cases except the following: In $B_{n}, A_{n j}=g^{n i} / 2,1 \leqq j \leqq n$; in $C_{n}, A_{i j}=g^{i j} / 2,1 \leqq i \leqq n-1,1 \leqq j \leqq n$; in $G_{2}, A_{2 j}=g^{2 j} / 3, j=1,2$; in $F_{4}, A_{i j}=g^{i j} / 2,1 \leqq j \leqq 4, i=3,4$. The inverse matrix $\left(B_{i j}\right)$ to $\left(A_{i j}\right)$ coincides with Dynkin's matrix $\left(g_{\alpha \beta}\right)$ except as follows: In $B_{n}, B_{i n}=2 g_{i n}, 1 \leqq i \leqq n$; in $C_{n}, B_{i j}=2 g_{i j}, 1 \leqq i \leqq n, 1 \leqq j \leqq n-1 ;$ in $G_{2}, B_{i 2}$ $=3 g_{i 2}, i=1,2$; in $F_{4}, B_{i j}=2 g_{i j}, 1 \leqq i \leqq 4, j=3,4$.

In any case, we have $e_{\alpha_{i}} \sigma \tau^{-1}=\mu_{i} e_{\beta_{i}}$ and $e_{-\alpha_{i}} \sigma \tau^{-1}=\nu_{i} e_{-\beta_{i}}, 1 \leqq i \leqq r$, where $\mu_{i} \neq 0, \nu_{i} \neq 0 .\left[e_{-\alpha_{i}} e_{\alpha_{i}}\right] \sigma \tau^{-1}=h_{i} \sigma \tau^{-1}$ and

$$
\alpha_{i \phi}\left(h_{i} \sigma \tau^{-1}\right)=\alpha_{i} \sigma \tau^{-1}\left(h_{i} \sigma \tau^{-1}\right)=\alpha_{i}\left(h_{i}\right)=2 .
$$

Moreover

$$
\left[e_{-\alpha_{i}} e_{\alpha_{i}}\right] \sigma \tau^{-1}=\left[e_{-\alpha_{i}} \sigma \tau^{-1}, e_{\alpha_{i}} \sigma \tau^{-1}\right]=\nu_{i} \mu_{i}\left[e_{-\beta_{i}} e_{\beta_{i}}\right] \in\left[\mathcal{R}_{-\beta_{i}} \ell_{\beta_{i}}\right]
$$

Hence

$$
\left[e_{-\alpha_{i}} e_{\alpha_{i}}\right] \sigma \tau^{-1}=h_{i \phi}=\left[e_{-\beta_{i}} e_{\beta_{i}}\right]=\nu_{i} \mu_{i}\left[e_{-\beta_{i}} e_{\beta_{i}}\right],
$$

$\nu_{i} \mu_{i}=1, \nu_{i}=\mu_{i}^{-1}$. Thus we see that $\omega=\sigma \tau^{-1}$ is completely determined on the $e_{-\alpha_{i}}$ by the nonzero scalars $\mu_{1}, \cdots, \mu_{r}$. Since the $e_{\alpha_{i}}$ and the $e_{-\alpha_{i}}$ generate $\mathbb{R}$ (by Lemma 1 above, (xii), (xiii) and Lemma 23 of (C)), $\omega$ is completely determined on $\&$ by the nonzero scalars $\mu_{1}, \cdots, \mu_{r}$. Summarizing, we have:

Lemma 6. Let $\omega$ be an automorphism mapping $\mathfrak{S}$ onto $\mathfrak{S}$ and such that $\alpha_{i}^{\omega}=\alpha_{i \phi}, 1 \leqq i \leqq r$, where $\phi \in \Sigma_{r}$. Then $\omega$ is completely determined by the permutation $\phi$ and the scalars $\mu_{1}, \cdots, \mu_{r}$.

IV. Automorphisms leaving a Cartan subalgebra fixed. We begin by investigating more closely the invariant automorphisms $\tau=T(\lambda, i)$ for $\lambda \neq 0$. We have

$$
\begin{aligned}
e_{\alpha_{i}} \tau & =e_{\alpha_{i}} B_{i}\left(-\lambda^{-1}\right) A_{2}(\lambda)=\left(e_{\alpha_{i}}+\lambda^{-1} h_{\imath}-\lambda^{-2} e_{-\alpha_{i}}\right) A_{i}(\lambda) \\
& =e_{\alpha_{i}}+\lambda^{-1} h_{i}-2 e_{\alpha_{i}}-\lambda^{-2} e_{-\alpha_{i}}-\lambda^{-1} h_{i}+e_{\alpha_{i}}=-\lambda^{-2} e_{-\alpha}, \\
e_{-\alpha_{i}} \tau & =\left(e_{-\alpha_{i}}+\lambda h_{i}-\lambda^{2} e_{\alpha_{i}}\right) B_{i}\left(-\lambda^{-1}\right) A_{i}(\lambda) \\
& =\left(e_{-\alpha_{i}}+\lambda h_{i}-2 e_{-\alpha_{i}}-\lambda^{2} e_{\alpha_{i}}-\lambda h_{i}+e_{-\alpha_{i}}\right) A_{i}(\lambda) \\
& =-\lambda^{2} e_{\alpha_{i}} A_{i}(\lambda)=-\lambda^{2} e_{\alpha_{i}} .
\end{aligned}
$$


Let $\beta$ be a root, $\beta \neq \pm \alpha_{i}, \beta-\alpha_{i}$ not a root, $\beta+\alpha_{i}$ a root.

CASE 1. $A_{\beta, \alpha_{i}}=-1$. Here $e_{\beta} \tau$ belongs to the $\operatorname{root} \beta^{r}=\beta S_{i}=\beta+\alpha_{i} ; \beta+2 \alpha_{i}$ is not a root, and we have

$$
\begin{aligned}
e_{\beta} \tau= & e_{\beta} A_{i}(\lambda) B_{i}\left(-\lambda^{-1}\right) A_{i}(\lambda)=\left(e_{\beta}+\lambda N_{\beta, \alpha_{i}} e_{\beta+\alpha_{i}}\right) B_{i}\left(-\lambda^{-1}\right) A_{i}(\lambda) \\
= & \left(e_{\beta}+\lambda N_{\beta, \alpha_{i}} e_{\beta+\alpha_{i}}-N_{\beta, \alpha_{i}} N_{\beta+\alpha_{i},-\alpha_{i}} e_{\beta}\right) A_{i}(\lambda) \\
= & \left(1-N_{\beta, \alpha_{i}} N_{\beta+\alpha_{i}-\alpha_{i}}\right) e_{\beta} \\
& +\lambda\left(1-N_{\beta, \alpha_{i}} N_{\beta+\alpha_{i},-\alpha_{i}}\right) N_{\beta, \alpha_{i}} e_{\beta+\alpha_{i}}+\lambda N_{\beta, \alpha_{i}} e_{\beta+\alpha_{i}} .
\end{aligned}
$$

Since $e_{\beta} \tau \in \Omega_{\beta+\alpha_{i}}$, the coefficient $1-N_{\beta, \alpha_{i}} N_{\beta+\alpha_{i},-\alpha_{i}}$ of $e_{\beta}$ must be zero; that is,

$$
N_{\beta, \alpha_{i}} N_{\beta+\alpha_{i},-\alpha_{i}}=1
$$

and

$$
e_{\beta} \tau=\lambda N_{\beta, \alpha_{i}} e_{\beta+\alpha_{i}} .
$$

CASE 2. $A_{\beta, \alpha_{i}}=-2$. Here $e_{\beta} \tau$ belongs to $\beta S_{i}=\beta+2 \alpha_{i}, \beta+3 \alpha_{i}$ is not a root, and

$$
\begin{aligned}
e_{\beta} \tau= & \left(e_{\beta}+\lambda N_{\beta, \alpha_{i}} e_{\beta+\alpha_{i}}+\frac{\lambda^{2}}{2 !} N_{\beta, \alpha_{i}} N_{\beta+\alpha_{i}, \alpha_{i}} e_{\beta+2 \alpha_{i}}\right) B_{i}\left(-\lambda^{-1}\right) A_{i}(\lambda) \\
= & \left(e_{\beta}+\lambda N_{\beta, \alpha_{i}} e_{\beta+\alpha_{i}}-N_{\beta, \alpha_{i}} N_{\beta+\alpha_{i},-\alpha_{i}} e_{\beta}+\frac{\lambda^{2}}{2 !} N_{\beta, \alpha_{i}} N_{\beta+\alpha_{i}, \alpha_{i}} e_{\beta+2 \alpha_{i}}\right. \\
& -\frac{\lambda}{2 !} N_{\beta, \alpha_{i}} N_{\beta+\alpha_{i}, \alpha_{i}} N_{\beta+2 \alpha_{i},-\alpha_{i}} e_{\beta+\alpha_{i}} \\
& \left.+\frac{1}{2 !^{2}} N_{\beta, \alpha_{i}} N_{\beta+\alpha_{i}, \alpha_{i}} N_{\beta+2 \alpha_{i},-\alpha_{i}} N_{\beta+\alpha_{i},-\alpha_{i}} e_{\beta}\right) A_{i}(\lambda)
\end{aligned}
$$

The coefficient of $e_{\beta}$ in $e_{\beta} \tau$, when the latter is written as a linear combination of root vectors, will be the same as that in $e_{\beta} A_{i}(\lambda) B_{i}\left(-\lambda^{-1}\right)$, namely

$$
1-N_{\beta, \alpha_{i}} N_{\beta+\alpha_{i},-\alpha_{i}}+\frac{1}{2 !^{2}} N_{\beta, \alpha_{i}} N_{\beta+\alpha_{i}, \alpha_{i}} N_{\beta+2 \alpha_{i},-\alpha_{i}} N_{\beta+\alpha_{i},-\alpha},
$$

and therefore this will be zero. Therefore

$$
\begin{aligned}
e_{\beta} \tau= & \left(\lambda N_{\beta, \alpha_{i}}\left(1-\frac{1}{2 !} N_{\beta+\alpha_{i}, \alpha_{i}} N_{\beta+2 \alpha_{i},-\alpha_{i}}\right) e_{\beta+\alpha_{i}}\right. \\
& \left.+\frac{\lambda^{2}}{2 !} N_{\beta, \alpha_{i}} N_{\beta+\alpha_{i}, \alpha_{i}} e_{\beta+2 \alpha_{i}}\right) A_{i}(\lambda)
\end{aligned}
$$

But now, as above, the coefficient of $e_{\beta+\alpha_{i}}$ in this expression must be zero, i.e. 


$$
N_{\beta+\alpha_{i}, \alpha_{i}} N_{\beta+2 \alpha_{i},-\alpha_{i}}=2 .
$$

From the fact that (12) is zero, we now conclude, using (13),

$$
N_{\beta, \alpha_{i}} N_{\beta+\alpha_{i},-\alpha_{i}}=2 \text {, }
$$

and $e_{\beta} \tau$ is $\left(\lambda^{2} / 2\right.$ !) $N_{\beta, \alpha_{i}} N_{\beta+\alpha_{i}, \alpha_{i}} e_{\beta+2 \alpha_{i}} A_{i}(\lambda)$; that is,

$$
e_{\beta} \tau=\frac{\lambda^{2}}{2 !} N_{\beta, \alpha_{i}} N_{\beta+\alpha_{i}, \alpha_{i}} e_{\beta+2 \alpha_{i}} .
$$

CAse 3. $A_{\beta, \alpha_{i}}=-3$. Here $e_{\beta} \tau$ belongs to $\beta+3 \alpha_{i}, \beta+4 \alpha_{i}$ is not a root, and

$$
\begin{aligned}
e_{\beta} \tau= & \left(e_{\beta}+\lambda N_{\beta, \alpha_{i}} e_{\beta+\alpha_{i}}+\frac{\lambda^{2}}{2 !} N_{\beta, \alpha_{i}} N_{\beta+\alpha_{i}, \alpha_{i}} e_{\beta+2 \alpha_{i}}\right. \\
& \left.+\frac{\lambda^{3}}{3 !} N_{\beta, \alpha_{i}} N_{\beta+\alpha_{i}, \alpha_{i}} N_{\beta+2 \alpha_{i}, \alpha_{i}} e_{\beta+3 \alpha_{i}}\right) B_{i}(-\lambda)^{-1} A_{i}(\lambda) \\
= & \left(e_{\beta}+\lambda N_{\beta, \alpha_{i}} e_{\beta+\alpha_{i}}-N_{\beta, \alpha_{i}} N_{\beta+\alpha_{i},-\alpha_{i}} e_{\beta}\right. \\
& +\frac{\lambda^{2}}{2 !} N_{\beta, \alpha_{i}} N_{\beta+\alpha_{i}, \alpha_{i}} e_{\beta+2 \alpha_{i}}-\frac{\lambda}{2 !} N_{\beta, \alpha_{i}} N_{\beta+\alpha_{i}, \alpha} N_{\beta+2 \alpha_{i},-\alpha_{i}} e_{\beta+\alpha} \\
& +\frac{1}{2 !^{2}} N_{\beta, \alpha_{i}} N_{\beta+\alpha_{i}, \alpha_{i}} N_{\beta+2 \alpha_{i},-\alpha_{i}} N_{\beta+\alpha_{i},-\alpha_{i}} e_{\beta} \\
& +\frac{\lambda^{3}}{3 !} N_{\beta, \alpha_{i}} N_{\beta+\alpha_{i}, \alpha_{i}} N_{\beta+2 \alpha_{i}, \alpha_{i}} e_{\beta+3 \alpha_{i}} \\
& -\frac{\lambda^{2}}{3 !} N_{\beta, \alpha_{i}} N_{\beta+\alpha_{i}, \alpha_{i}} N_{\beta+2 \alpha_{i}, \alpha_{i}} N_{\beta+3 \alpha_{i},-\alpha_{i}} e_{\beta+2 \alpha_{i}} \\
& +\frac{\lambda}{2 ! 3 !} N_{\beta, \alpha_{i}} N_{\beta+\alpha_{i}, \alpha_{i}} N_{\beta+2 \alpha_{i}, \alpha_{i}} N_{\beta+3 \alpha_{i},-\alpha_{i}} N_{\beta+2 \alpha_{i},-\alpha_{i}} e_{\beta+\alpha} \\
& \left.-\frac{1}{3 !^{2}} N_{\beta, \alpha_{i}} N_{\beta+\alpha_{i}, \alpha_{i}} N_{\beta+2 \alpha_{i}, \alpha_{i}} N_{\beta+3 \alpha_{i},-\alpha_{i}} N_{\beta+2 \alpha_{i},-\alpha_{i}} N_{\beta+\alpha_{i},-\alpha_{i}} e_{\beta}\right) A_{i}(\lambda) .
\end{aligned}
$$

As in Cases 1 and 2, we find from observing in turn that the coefficients of $e_{\beta}, e_{\beta+\alpha_{i}}, e_{\beta+2 \alpha_{i}}$ in $e_{\beta} \tau$, hence in $e_{\beta} A_{i}(\lambda) B_{i}\left(-\lambda^{-1}\right)$, must be zero, that

$$
\begin{aligned}
N_{\beta+2 \alpha_{i}, \alpha_{i}} N_{\beta+3 \alpha_{i},-\alpha_{i}} & =3, \\
N_{\beta+\alpha_{i}, \alpha_{i}} N_{\beta+2 \alpha_{i},-\alpha_{i}} & =4, \\
N_{\beta, \alpha_{i}} N_{\beta+\alpha_{i},-\alpha_{i}} & =3,
\end{aligned}
$$

and finally that

$$
e_{\beta} \tau=\frac{\lambda^{3}}{3 !} N_{\beta, \alpha_{i}} N_{\beta+\alpha_{i}, \alpha_{i}} N_{\beta+2 \alpha_{\imath}, \alpha_{i}} e_{\beta+3 \alpha_{i}}
$$


Now let $\gamma \neq \pm \alpha_{i}$ be a root such that $\gamma+\alpha_{i}$ is not a root. If $\gamma-\alpha_{i}$ is not a root, then $e_{\gamma} \tau=e_{\gamma} T(\lambda, i)=e_{\gamma}$. Otherwise, we have three cases:

CASE $1^{\prime} . A_{\gamma, \alpha_{i}}=1$. Here $\gamma^{\tau}=\gamma-\alpha_{i}, e_{\gamma} \tau \in \mathbb{R}_{\gamma-\alpha_{i}}, \gamma-2 \alpha_{i}$ is not a root, and

$$
\begin{aligned}
e_{\gamma} \tau & =e_{\gamma} A_{i}(\lambda) B_{i}\left(-\lambda^{-1}\right) A_{i}(\lambda)=e_{\gamma} B_{i}\left(-\lambda^{-1}\right) A_{i}(\lambda) \\
& =\left(e_{\gamma}-\lambda^{-1} N_{\gamma,-\alpha_{i}} e_{\gamma-\alpha_{i}}\right) A_{i}(\lambda) \\
& =-\lambda^{-1} N_{\gamma,-\alpha_{i}} e_{\gamma-\alpha_{i}}+\left(1-N_{\gamma,-\alpha_{i}} N_{\gamma-\alpha_{i}, \alpha_{i}}\right) e_{\gamma} .
\end{aligned}
$$

As in Case 1, we have

$$
N_{\gamma,-\alpha_{i}} N_{\gamma-\alpha_{\imath}, \alpha_{i}}=1 \text {, }
$$

and

$$
e_{\gamma} \tau=-\lambda^{-1} N_{\gamma,-\alpha_{i}} e_{\gamma-\alpha_{i}} .
$$

CASE $2^{\prime} . A_{\gamma, \alpha_{i}}=2$. The procedures established in the preceding cases yield

$$
\begin{aligned}
N_{\gamma-\alpha_{i},-\alpha_{i}} N_{\gamma-2 \alpha_{i}, \alpha_{i}} & =2, \\
N_{\gamma,-\alpha_{i}} N_{\gamma-\alpha_{i}, \alpha_{i}} & =2,
\end{aligned}
$$

and

$$
e_{\gamma} \tau=\frac{\lambda^{-2}}{2 !} N_{\gamma,-\alpha_{i}} N_{\gamma-\alpha_{i},-\alpha_{i}} e_{\gamma-2 \alpha_{i}} .
$$

CASE $3^{\prime} . A_{\gamma, \alpha_{i}}=3$. As in previous cases, we find

$$
\begin{aligned}
N_{\gamma-2 \alpha_{i},-\alpha_{i}} N_{\gamma-3 \alpha_{i}, \alpha_{i}} & =3, \\
N_{\gamma-\alpha_{i},-\alpha_{i}} N_{\gamma-2 \alpha_{i}, \alpha_{i}} & =4, \\
N_{\gamma,-\alpha_{i}} N_{\gamma-\alpha_{i}, \alpha_{i}} & =3,
\end{aligned}
$$

and

$$
e_{\gamma} \tau=\frac{-\lambda^{-3}}{3 !} N_{\gamma,-\alpha_{i}} N_{\gamma-\alpha_{i},-\alpha_{i}} N_{\gamma-2 \alpha_{i},-\alpha_{i}} e_{\gamma-3 \alpha_{i}} .
$$

In particular, the primed cases apply when $\beta$ is as in Cases 1, 2, 3 and $\gamma=\beta S_{i}=\beta-A_{\beta, \alpha_{i}} \alpha_{i}$. Then $A_{\gamma, \alpha_{i}}=A_{\beta, \alpha_{i}}-A_{\beta, \alpha_{i}} A_{\alpha_{i}, \alpha_{i}}=A_{\beta, \alpha_{i}}-2 A_{\beta, \alpha_{i}}$ $=-A_{\beta, \alpha_{i}}$. Thus if $\beta$ is as in Case $1,2,3$, then $\gamma=\beta S_{i}$ is as in Case $1^{\prime}, 2^{\prime}, 3^{\prime}$, respectively, and the Equations (20), (22), (23), (25), (26), (27) are corollaries of the respective Equations (10), (14), (13), (18), (17), (16).

Now let $\omega=U(\lambda, i)=T(\lambda, i) T(-1, i)$. Then if $\alpha_{i}(h)=0$, we have $h \omega=h$, and $h_{i} \omega=-h_{i} T(-1, i)=h_{i}$. Thus $\omega$ is the identity on $\mathfrak{S}$. Moreover, $\alpha^{\omega}$ $=\left(\alpha S_{i}\right) S_{i}=\alpha S_{t}^{2}=\alpha$ for all roots $\alpha$. By Lemma $6, \omega$ is completely determined by its effect on the $e_{\alpha_{j}}, 1 \leqq j \leqq r$. By (8) and (9), we have

$$
\begin{aligned}
& e_{\alpha_{i}} \omega=e_{\alpha_{i}} T(\lambda, i) T(-1, i)=-\lambda^{-2} e_{-\alpha_{i}} T(-1, i)=\lambda^{-2} e_{\alpha_{i}}: \\
& e_{\alpha_{i}} \omega=\lambda^{-2} e_{\alpha_{i}}=\lambda^{-A_{i i}} e_{\alpha_{i}} .
\end{aligned}
$$


If $\beta \neq \pm \alpha_{i}$, and if $\beta-\alpha_{i}$ is not a root, we have:

For $A_{\beta, \alpha_{i}}=0$ :

$$
e_{\beta} \omega=e_{\beta}=\lambda^{-A_{\beta, \alpha_{i}} e_{\beta}}
$$

by the remark following (19).

For $A_{\beta, \alpha_{i}}=-1$ :

$$
e_{\beta} \omega=\lambda N_{\beta, \alpha_{i}} e_{\beta+\alpha_{i}} T(-1, i)=\lambda N_{\beta, \alpha_{i}} N_{\beta+\alpha_{i},-\alpha_{i}} e_{\beta}=\lambda e_{\beta},
$$

by (11), (21), and (10). Thus

$$
e_{\beta} \omega=\lambda e_{\beta}=\lambda^{-A_{\beta, \alpha_{i}} e_{\beta} .}
$$

For $A_{\beta, \alpha_{i}}=-2$ :

$$
\begin{aligned}
e_{\beta} \omega & =\frac{\lambda^{2}}{2 !} N_{\beta, \alpha_{i}} N_{\beta+\alpha_{i}, \alpha_{i}} e_{\beta+2 \alpha_{i}} T(-1, i) \\
& =\frac{\lambda^{2}}{2 !^{2}} N_{\beta, \alpha_{i}} N_{\beta+\alpha_{i}, \alpha_{i}} N_{\beta+2 \alpha_{i},-\alpha_{i}} N_{\beta+\alpha_{i},-\alpha_{i}} e_{\beta},
\end{aligned}
$$

by (15) and (24). Now, applying (13) and (14), we have

$$
e_{\beta} \omega=\lambda^{2} e_{\beta}=\lambda^{-A_{\beta, \alpha_{i}} e_{\beta} .}
$$

For $A_{\beta, \alpha_{i}}=-3$ :

$$
\begin{aligned}
e_{\beta} \omega & =\frac{\lambda^{3}}{3 !} N_{\beta, \alpha_{i}} N_{\beta+\alpha_{i}, \alpha_{i}} N_{\beta+2 \alpha_{i}, \alpha_{i}} e_{\beta+3 \alpha_{i}} T(-1, i) \\
& =\frac{\lambda^{3}}{3 !^{2}} N_{\beta, \alpha_{i}} N_{\beta+\alpha_{i}, \alpha_{i}} N_{\beta+2 \alpha_{i}, \alpha_{i}} N_{\beta+3 \alpha_{i},-\alpha_{i}} N_{\beta+2 \alpha_{i},-\alpha_{i}} N_{\beta+\alpha_{i},-\alpha_{i}} e_{\beta},
\end{aligned}
$$

by (19) and (28). Using (16), (17), and (18), we obtain

$$
e_{\beta} \omega=\lambda^{3} e_{\beta}=\lambda^{-A_{\beta, \alpha_{i}} e_{\beta} .}
$$

From (29)-(33), we see that for all $j, 1 \leqq j \leqq r$,

$$
e_{\alpha_{j}} \omega=\lambda^{-A_{j i}} e_{\alpha_{j}} .
$$

Now if $\lambda_{1}, \cdots, \lambda_{r}$ are nonzero elements of $\mathfrak{F}$, and if $\eta=U\left(\lambda_{1}, 1\right) \cdots$ $U\left(\lambda_{r}, r\right)$, we have $h \eta=h$ for all $h \in \mathfrak{S}$, and

$$
e_{\alpha_{j}} \eta=\left(\prod_{i=1}^{r} \lambda_{i}^{-A_{j i}}\right) e_{\alpha_{j}}, \quad 1 \leqq j \leqq r .
$$

The determinant $d=\left|A_{i j}\right|$ has been seen in (C) (Lemma 11) to be $r+1$, if $\alpha_{1}, \cdots, \alpha_{r}$ is of type $A_{r} ; 2$, if $\alpha_{1}, \cdots, \alpha_{r}$ is of type $B_{r}, C_{r}$ or $E_{7} ; 4$, if $\alpha_{1}, \cdots, \alpha_{r}$ is of type $D_{r} ; 3$, if $\alpha_{1}, \cdots, \alpha_{r}$ is of type $E_{6} ; 1$, if $\alpha_{1}, \cdots, \alpha_{r}$ is of type $G_{2}, F_{4}$ or $E_{8}$. Hence there are rational integers $B_{i k}, 1 \leqq i, k \leqq r$, (which can be found 
from Table 2 of [6]), such that

$$
\sum_{i=1}^{r} A_{j i} B_{i k}=\delta_{j k} d=\sum_{i=1}^{r} B_{j i} A_{i k}
$$

$1 \leqq j, k \leqq r$. Thus if $\lambda_{i}=\coprod_{k=1}^{r} \rho_{k}^{-B_{i k}}$, (35) becomes

$$
\begin{aligned}
e_{\alpha_{j} \eta} & =\prod_{i=1}^{r}\left(\prod_{k=1}^{r} \rho^{-B_{i k}}\right)^{-A_{j i}} e_{\alpha_{j}} \\
& =\left(\prod_{i=1}^{r} \prod_{k=1}^{r} \rho_{k}^{A_{j i} B_{i k}}\right) e_{\alpha_{j}} \\
& =\left(\prod_{k=1}^{r} \prod_{i=1}^{r} \rho^{A_{j i} B_{i k}}\right) e_{\alpha_{j}}=\left(\prod_{k=1}^{r}{\left.\stackrel{\rho_{k}^{r}}{\Sigma_{i-A_{j i} B_{i k}}}\right) e_{\alpha_{j}}}=\left(\prod_{k=1}^{r} \rho_{k}^{\delta_{j k d}}\right) e_{\alpha_{j}}=\rho_{\rho_{j}}^{d} e_{\alpha_{j}},\right.
\end{aligned}
$$$$
1 \leqq j \leqq r
$$

If $\sigma$ is any automorphism of $\mathfrak{R}$ such that $\mathfrak{S c} \sigma=\mathfrak{S}$ and such that $\alpha_{j}^{\sigma}=\alpha_{j}$, we have seen in Lemma 6 that $h \sigma=h$ for all $h \in \mathfrak{S}$, and that $\sigma$ is completely determined by the scalars $\mu_{j} \neq 0$ such that $e_{\alpha_{j}} \sigma=\mu_{j} e_{\alpha_{j}}, 1 \leqq j \leqq r$. But if each $\mu_{j}$ is a $d$ th power in $\mathfrak{F}$, say $\mu_{j}=\rho_{j}^{d}, 1 \leqq j \leqq r$, and if we set

$$
\lambda_{i}=\prod_{k=1}^{r} \rho_{k}^{-B_{i k}}, \quad 1 \leqq i \leqq r,
$$

and $\eta=U\left(\lambda_{1}, 1\right) \cdots U\left(\lambda_{r}, 1\right)$, then we see by (36) that $\eta$ is an invariant automorphism which coincides with $\sigma$ on $\mathfrak{S}$ and on $e_{\alpha_{1}}, \cdots, e_{\alpha_{r}}$. Consequently $\sigma=\eta$ is an invariant automorphism. We have thus proved the following:

THEOREM 4. Let $\mathfrak{R}$ be a simple Lie algebra of classical type, $\mathfrak{S}$ a standard Cartan subalgebra. Let $\alpha_{1}, \cdots, \alpha_{r}$ be a fundamental system of roots relative to $\mathfrak{S}$ with matrix $\left(A_{i j}\right)$ having as determinant the natural number $d$. Let $\sigma$ be an automorphism of $\mathbb{R}$ such that $\mathfrak{S} \sigma=\mathfrak{S}$ and $\alpha_{i}^{\sigma}=\alpha_{i}, 1 \leqq i \leqq r$. Suppose further that $e_{\alpha_{i}} \sigma=\mu_{i} e_{\alpha_{i}}, 1 \leqq i \leqq r$, where each $\mu_{i}$ is a dth power in $\mathfrak{F}$. Then $\sigma$ is an invariant automorphism, and an explicit expression for $\sigma$ may be found by the fact that $\sigma=\eta$, where $\eta$ is constructed as in (37), with the $U\left(\lambda_{i}, i\right)$ as defined above.

Corollary 1. Let $\mathfrak{F}$ be algebraically closed, and let $\mathbb{i}, \mathfrak{S}$ and $\alpha_{1}, \cdots, \alpha_{r}$ be as in the statement of Theorem 4. Let $\sigma$ be any automorphism of \& such that $\mathfrak{S} \sigma=\mathfrak{S}$ and $\alpha_{i}^{\sigma}=\alpha_{i}, 1 \leqq i \leqq r$. Then $\sigma$ is an invariant automorphism. Moreover, if $\mu_{1}, \cdots, \mu_{r}$ are any nonzero scalars and $e_{\alpha_{i}}$ any nonzero elements of $\Re_{\alpha_{i}}, 1 \leqq i \leqq r$, there is an invariant automorphism $\eta$ such that $h \eta=h$ for all $h \in \mathfrak{S}$ and $e_{\alpha_{i}} \eta=\mu_{i} e_{\alpha_{i}}, 1 \leqq i \leqq r$.

Corollary 2. Let $\mathfrak{R}, \mathfrak{S}, \alpha_{1}, \cdots, \alpha_{r}$ be as in the statement of Theorem 4 , and suppose that \& (or $\left.\alpha_{1}, \cdots, \alpha_{r}\right)$ is of one of the types $G_{2}, F_{4}, E_{8}$. Let $\sigma$ be any 
automorphism of $\mathfrak{R}$ such that $\mathfrak{S} \sigma=\mathfrak{S}$ and $\alpha_{i}^{\sigma}=\alpha_{i}, 1 \leqq i \leqq r$. Then $\sigma$ is an invariant automorphism; in fact, the condition $\alpha_{i}^{\sigma}=\alpha_{i}$ can be omitted from the hypotheses without changing the conclusion.

Proof. In these three cases, $d=1$; here $\mathfrak{F}$ contains $d$ th roots of all elements. Thus the first part of the corollary follows from Theorem 4 . If $\mathfrak{S} \sigma=\mathfrak{F}$, then by Theorem 3 , we have $\mathfrak{S} \sigma \tau^{-1}=\mathfrak{H}$, and the $\alpha_{\imath}^{\sigma \tau^{-1}}$ are a permutation of the $\alpha_{i}$, for some invariant automorphism $\tau$. By the remarks following Theorem 3, this can only be the identity permutation in these cases. Hence $\sigma \tau^{-1}$ is an invariant automorphism by the first part of Corollary 2, and therefore $\sigma$ is an invariant automorphism.

V. The algebraically closed case. Let $\mathfrak{R}, \mathfrak{S}$, and $\alpha_{1}, \cdots, \alpha_{r}$ be as above, and let the base field $\mathfrak{F}$ be algebraically closed. Let $\sigma$ be an automorphism of \&. Then by Theorem 1 there is an invariant automorphism $\tau$ such that $\mathfrak{S} \sigma \tau^{-1}=\mathfrak{S}$. Then $\sigma \tau^{-1}$ maps $\alpha_{1}, \cdots, \alpha_{r}$ onto another fundamental system with respect to $\mathfrak{S}$, and by Theorem 3 there is an invariant automorphism $\omega$ of $\mathbb{R}$ such that $\mathfrak{S} \sigma \tau^{-1} \omega^{-1}=\mathfrak{S}$ and such that $\sigma \tau^{-1} \omega^{-1}$ permutes the system $\alpha_{1}, \cdots, \alpha_{r}$. Let $\phi$ be that permutation of the indices $1, \cdots, r$ such that $\alpha_{i} \sigma \tau^{-1} \omega^{-1}=\alpha_{i \phi}, 1 \leqq i \leqq r$. Let $0 \neq e_{\alpha_{i}} \in \mathfrak{R}_{\alpha_{i}}, 1 \leqq i \leqq r$, and let $e_{\alpha_{i}} \sigma \tau^{-1} \omega^{-1}=\mu_{i} e_{\alpha_{i \phi}}$ $1 \leqq i \leqq r$. Then by Corollary 1 , there is an invariant automorphism $\eta$ such that $\mathfrak{S} \sigma \tau^{-1} \omega^{-1} \eta^{-1}=\mathfrak{S}$ and such that $e_{\alpha_{i \phi}} \eta=\mu_{i} e_{\alpha_{i \phi}}, 1 \leqq i \leqq r$, or $\mu_{i} e_{\alpha_{i \phi}} \eta^{-1}=e_{\alpha_{i \phi}}$; that is, $e_{\alpha_{i}} \sigma \tau^{-1} \omega^{-1} \eta^{-1}=e_{\alpha_{i \phi}}, 1 \leqq i \leqq r$. Thus $\sigma \tau^{-1} \omega^{-1} \eta^{-1}$ is an automorphism of $\mathfrak{R}$ mapping $\mathfrak{S}$ onto $\mathfrak{S}$ and $e_{\alpha_{i}}$ onto $e_{\alpha_{i \phi}}, 1 \leqq i \leqq r$. If $\phi$ is the identity permutation, we see by Lemma 6 that $\sigma \tau^{-1} \omega^{-1} \eta^{-1}$ is the identity automorphism, and, by III, this is necessarily the case unless $\&$ is of type $A_{r}(r \geqq 2), D_{r}(r \geqq 4)$, or $E_{6}$. Thus $\sigma=\eta \omega \tau$ is an invariant automorphism except in these cases, and we have proved:

THEOREM 5. Let $\mathfrak{F}$ be algebraically closed, and suppose that $\&$ is not of type $A_{r}(r \geqq 2)$, of type $D_{r}(r \geqq 4)$ or of type $E_{6}$. Then every automorphism of $\mathbb{R}$ is an invariant automorphism.

In general, let $\Im$ be the group of invariant automorphisms, and consider the automorphism $\sigma \tau^{-1} \omega^{-1} \eta^{-1}$ mapping $\mathfrak{S}$ onto $\mathfrak{S}$ and $e_{\alpha_{i}}$ onto $e_{\alpha_{i \phi}}, 1 \leqq i \leqq r$. Such an automorphism exists by the proof of Theorem 9 of [13] and is unique by Lemma 6 . Let us denote this automorphism by $\zeta(\phi)$. Then $\sigma=\zeta(\phi) \eta \omega \tau \in \zeta(\phi) \Im$. In case $\mathcal{R}$ is of type $A_{r}(r \geqq 2), D_{r}(r \geqq 5)$, or $E_{6}$, there are only two possible values for $\phi$, consequently for $\zeta(\phi)$; hence $\Im$ is a subgroup of index at most two in the full automorphism group $\mathfrak{A}$. In case $\mathfrak{R}$ is of type $D_{4}, \mathfrak{Y}$ is a subgroup of $\mathfrak{A}$ of index at most 6 . In any case, $\mathfrak{R}$ is readily seen to be a normal subgroup of $\mathfrak{A}$. With the exception of the case where $\mathfrak{R}$ is of type $E_{6}$, it is known that the indices are precisely 2 and 6 , respectively, and in case $\mathfrak{l}$ is of type $E_{6}$, the results in the complex case indicate that the index is 2 . The author proposes to elaborate on the results for the individual simple 
algebras in a sequel to this paper. Our results for the algebras not covered by Theorem 5 are as follows:

TheOREM 6. If $\mathfrak{F}$ is algebraically closed and if $\mathfrak{R}$ is of type $A_{r}(r \geqq 2)$ or of type $D_{r}(r \geqq 5)$, then the invariant automorphisms form a subgroup of index 2 in the full automorphism group. If $\mathbb{R}$ is of type $D_{4}$, the invariant automorphisms form a normal subgroup of index 6 in the full automorphism group, and the quotient group is the symmetric group on three letters. If $\&$ is of type $E_{6}$, the indicated index is 2 .

\section{BIBLIOGRAPHY}

1. E. Cartan, Les groupes projectifs qui ne laissent invariante aucune multiplicité plane, Bull. Soc. Math. France vol. 41 (1913) pp. 53-96.

2. C. Chevalley, Sur certains groupes simples, Tôhoku Math. J. vol. 7 (1955) pp. 14-66.

3. C. W. Curtis, Modular Lie algebras I, Trans. Amer. Math. Soc. vol. 82 (1956) pp. 160179.

4. - Modular Lie algebras II, Trans. Amer. Math. Soc. vol. 86 (1957) pp. 91-108.

5. E. B. Dynkin, The structure of semi-simple algebras, Amer. Math. Soc. Translations, Ser. 1, no. 17 .

6. - Semisimple subalgebras of semisimple Lie algebras, Amer. Math. Soc. Translations, Ser. 2, vol. 6, 1957, pp. 111-244.

7. F. Gantmacher, Canonical representation of automorphisms of a complex semi-simple Lie group, Mat. Sb. vol. 5 (47) (1939) pp. 101-146.

8. Harish-Chandra, On some applications of the universal enveloping algebra of a semi-simple Lie algebra, Trans. Amer. Math. Soc. vol. 70 (1951) pp. 28-96.

9. N. Jacobson, Simple Lie algebras over a field of characteristic zero, Duke Math. J. vol. 4 (1938) pp. 534-551.

10. - Cayley numbers and simple Lie algebras of type G, Duke Math. J. vol. 5 (1939) pp. 775-783.

11. - Classes of restricted Lie algebras of characteristic p, I, Amer. J. Math. vol. 63 (1941) pp. 481-515.

12. W. H. Mills, Classical type Lie algebras of characteristic 5 and 7, J. Math. Mech. vol. 6 (1957) pp. 559-566.

13. W. H. Mills and G. B. Seligman, Lie algebras of classical type, J. Math. Mech. vol. 6 (1957) pp. 519-548.

14. G. B. Seligman, On Lie algebras of prime characteristic, Memoirs Amer. Math. Soc., no. $19,1956$. 558.

15. —_ Some remarks on classical Lie algebras, J. Math. Mech. vol. 6 (1957) pp. 549-

16. Séminaire Sophus Lie, mimeographed notes, École Normale Supérieure, Paris, 19541955.

17. M. L. Tomber, Lie algebras of type F, Proc. Amer. Math. Soc. vol. 4 (1953) pp. 759-768.

18. H. Weyl, Theorie der Darstellung kontinuierlicher halbeinfacher Gruppen durch lineare Transformationen II, Math. Z. vol. 24 (1926) pp. 328-376.

19. E. Witt, Spiegelungsgruppen und Aufzählung halbeinfacher Liescher Ringe, Abh. Math. Sem. Univ. Hamburg vol. 14 (1941) pp. 289-337.

20. I. Satake, On a theorem of E. Cartan, J. Math. Soc. Japan vol. 2 (1951) pp. 284-305.

YALE UNIVERSITY,

New Haven, Connecricut 